\begin{tabular}{|c|c|}
\hline Title & Localization of Bott-Chern classes and Hermitian residues \\
\hline Author(s) & Correa, Mauricio, Jr.; Suwa, Tatsuo \\
\hline Citation & $\begin{array}{l}\text { Journal of the London Mathematical Society. Second series, 101(1), 349-372 } \\
\text { https://doi.org/10.1112/1ms.12273 }\end{array}$ \\
\hline Issue Date & $2020-02$ \\
\hline Doc URL & http:/hdl.handle.net/2115/76861 \\
\hline Rights & $\begin{array}{l}\text { This is the accepted version of the following article: Localization of Bott- Chern classes and Hermitian residues, which } \\
\text { has been published in final form at https://ondmathsoc.onlinelibrary.wiley .com/doi full/10.1112/1ms.12273. }\end{array}$ \\
\hline Type & article (author version) \\
\hline File Information & Bott-Chern(rev2).pdf \\
\hline
\end{tabular}

Instructions for use 


\title{
Localization of Bott-Chern classes and Hermitian residues
}

\author{
Maurício Corrêa Jr*and Tatsuo Suwa ${ }^{\dagger}$
}

\begin{abstract}
We develop a theory of Čech-Bott-Chern cohomology and in this context we naturally come up with the relative Bott-Chern cohomology. In fact Bott-Chern cohomology has two relatives and they all arise from a single complex. Thus we study these three cohomologies in a unified way and obtain a long exact sequence involving the three. We then study the localization problem of characteristic classes in the relative BottChern cohomology. For this we define the cup product and integration in our framework and we discuss local and global duality morphisms. After reviewing some materials on connections, we give a vanishing theorem relevant to our localization. With these, we prove a residue theorem for vector bundles admitting a Hermitian connection compatible with an action of the non-singular part of a singular distribution. As a typical case, we discuss the action of a distribution on the normal bundle of an invariant submanifold (the so-called Camacho-Sad action) and give a specific example.
\end{abstract}

Mathematics Subject Classification (2010): Primary 32A27, 32C35, 32S65, 53C56; Secondary 14C30, 53B35, 53C05, 57R20.

Keywords: Relative Bott-Chern cohomology, local duality morphism, metric connection, Bott type vanishing theorem, localization and Hermitian residue, singular holomorphic distribution.

\section{Introduction}

Čech-de Rham cohomology, particularly the relative version, together with its integration theory has been extensively used in the localization problem of characteristic classes. It started with the residue problem of singular holomorphic foliations (cf. [17], [18], [21]) and the theory was then transferred to the fixed point theory of discrete dynamics (cf. [1]). The philosophy behind is rather simple. Namely, once we have some kind of vanishing theorem on the non-singular part, certain characteristic classes are localized at the set of singular points and the localization gives rise to residues and the residue theorem via the Alexander duality. The relative easiness of computing the residues is another advantage of this method. The idea and the techniques turned out to be effective in many other problems, characteristic classes of singular varieties and localized intersection theory, to

\footnotetext{
* Supported by CAPES, CNPq and Fapesp

†Supported by JSPS grants no. 24540060 and no. $16 \mathrm{~K} 05116$
} 
name a few (cf. [10], [22]). A similar theory is developed for the Dolbeault complex, the relevant characteristic classes in this case being the Atiyah classes (cf. [2], [23], [24], [26]).

In this paper we study the localization problem in Bott-Chern cohomology. The cohomology, which was introduced in [8], refines both de Rham and Dolbeault cohomologies. It is a powerful tool in the study of non-Kähler manifolds (cf. [3], [4] and references therin) and is also related to arithmetic characteristic classes (cf. [11], [14]). We first develop a theory of Cech-Bott-Chern cohomology and the relative Bott-Chern cohomology naturally arises in this context. In fact Bott-Chern cohomology has some relatives, i.e., Aeppli cohomology and one more, and they all come from a single complex (cf. (2.1) below). This viewpoint allows us to deal with the three types of cohomologies in a unified way and leads to a long exact sequence involving the three (Theorem 3.26). As to the local and global dualities, we are mainly interested in the one between Bott-Chern and Aeppli. For this the cup product is defined on the cochain level and the integration is defined via Čech-de Rham cohomology.

In the de Rham case, the usual Chern-Weil theory of characteristic classes fits nicely into the theory with some modifications and, in the Dolbeault case, a similar strategy works by considering connections of type $(1,0)$. In the Bott-Chern case we impose one more condition on the connections, i.e., we require the connections to be Hermitian. We then define characteristic classes in the Cech-Bott-Chern cohomology using the BottChern form. The relevant vanishing theorem in our case is the one proved in [2] together with the Hermitian condition (Corollary 6.4). With these at hand, we have a general residue theorem for a holomorphic vector bundle admitting a Hermitian connection compatible with an action of the non-singular part of a singular distribution (Theorem 7.3).

Here is an outline of the paper. In Section 2, we recall the three types of cohomologies, including Bott-Chern and Aeppli, by considering the complex (2.1). We then recall, in Section 3, the theory of Čech cohomology of a complex of sheaves of differential forms and we discuss its relative version. These are applied to the three cases and we have the relative Bott-Chern, the relative Aeppli cohomologies and one more. As mentioned above, we give a long exact sequence relating these three cohomologies (Theorem 3.26). We also dicuss the relation with the Dolbeault case (Theorem 3.29). In order to state local and global duality morphisms, we discuss, in Section 4, the cup product in this cohomology theory, in particular the one between Bott-Chern and Aeppli. Then we discuss the integration theory mainly on Aeppli cohomology. In Section 5, we define the characteristic classes of a holomorphic vector bundle in Cech-Bott-Chern cohomology. The vanishing theorem we need is given in Section 6 (Corollary 6.4). It arises from an action of a non-singular distribution on a vector bundle and, as an example, we present the action on the normal bundle of an invariant submanifold of a distribution, the so-called Camacho-Sad action. In Section 7, we discuss localization theory of characteristic classes of vector bundles with actions of singular distributions and the associated Hermitian residues (Theorem 7.3). Finally we give an example in Section 8. It concerns with a Hopf surface with a fibration of elliptic curves on the projective line. We show that the Bott-Chern class of the pull-back of the hyperplane bundle is localized at one of the fibers.

Acknowledgment: The first named author is grateful to Hokkaido University for hospitality. The both authors would like to thank the referees for precious comments, in particular on Bott-Chern forms, which improved the presentation of the paper greatly. 


\section{Bott-Chern cohomology and its companions}

We list [3], [4] and [20] as references for this section.

Let $M$ denote a complex manifold of dimension $n$. Also let $\mathcal{E}^{r}$ and $\mathcal{E}^{p, q}$ denote the sheaves of $C^{\infty}$ differential $r$-forms and $(p, q)$-forms on $M$. For a sheaf $\mathcal{S}$ and an open set $U$ in $M$, we denote by $\mathcal{S}(U)$ the set of sections on $U$.

We have the decomposition

$$
\mathcal{E}^{r}=\bigoplus_{p+q=r} \mathcal{E}^{p, q}
$$

and accordingly the exterior derivative $d: \mathcal{E}^{r} \rightarrow \mathcal{E}^{r+1}$ has the decomposition $d=\partial+\bar{\partial}$ :

$$
\partial: \mathcal{E}^{p, q} \longrightarrow \mathcal{E}^{p+1, q}, \quad \bar{\partial}: \mathcal{E}^{p, q} \longrightarrow \mathcal{E}^{p, q+1} .
$$

Recall that the $r$-th de Rham cohomology $H_{d}^{r}(M)$ of $M$ is the $r$-th cohomology of the complex $\left(\mathcal{E}^{\bullet}(M), d\right)$ and the Dolbeault cohomology $H_{\bar{\partial}}^{p, q}(M)$ of type $(p, q)$ of $M$ is the $q$-th cohomology of the complex $\left(\mathcal{E}^{p, \bullet}(M), \bar{\partial}\right)$.

We set

$$
\mathcal{E}^{(p, q)+1}=\mathcal{E}^{p+1, q} \oplus \mathcal{E}^{p, q+1}
$$

and consider the complex

$$
\ldots \stackrel{d}{\longrightarrow} \mathcal{E}^{(p-2, q-2)+1} \stackrel{\bar{\partial}+\partial}{\longrightarrow} \mathcal{E}^{p-1, q-1} \stackrel{\bar{\partial} \partial}{\longrightarrow} \mathcal{E}^{p, q} \stackrel{d}{\longrightarrow} \mathcal{E}^{(p, q)+1} \stackrel{\bar{\partial}+\partial}{\longrightarrow} \mathcal{E}^{p+1, q+1} \stackrel{\bar{\partial} \partial}{\longrightarrow} \cdots .
$$

Bott-Chern cohomology: The Bott-Chern cohomology $H_{\mathrm{BC}}^{p, q}(M)$ of type $(p, q)$ of $M$ is the cohomology of the complex

$$
\mathcal{E}^{p-1, q-1}(M) \stackrel{\bar{\partial} \partial}{\longrightarrow} \mathcal{E}^{p, q}(M) \stackrel{d}{\longrightarrow} \mathcal{E}^{(p, q)+1}(M) .
$$

We have canonical morphisms

$$
H_{\mathrm{BC}}^{p, q}(M) \longrightarrow H_{\bar{\partial}}^{p, q}(M), \quad \bigoplus_{p+q=r} H_{\mathrm{BC}}^{p, q}(M) \longrightarrow H_{d}^{r}(M) .
$$

If $M$ is compact Kähler, for instance, the above morphisms are isomorphisms.

Aeppli cohomology: The Aeppli cohomology $H_{\mathrm{A}}^{p, q}(M)$ of type $(p, q)$ of $M$ is the cohomology of the complex

$$
\mathcal{E}^{(p-1, q-1)+1}(M) \stackrel{\bar{\partial}+\partial}{\longrightarrow} \mathcal{E}^{p, q}(M) \stackrel{\bar{\partial} \partial}{\longrightarrow} \mathcal{E}^{p+1, q+1}(M) .
$$

In particular, $H_{\mathrm{A}}^{n, n}(M)=H_{d}^{2 n}(M)$. We have canonical morphisms

$$
H_{\bar{\partial}}^{p, q}(M) \longrightarrow H_{\mathrm{A}}^{p, q}(M), \quad H_{d}^{r}(M) \longrightarrow \bigoplus_{p+q=r} H_{\mathrm{A}}^{p, q}(M) .
$$

Note that the differential $\partial: \mathcal{E}^{p, q}(M) \rightarrow \mathcal{E}^{p+1, q}(M)$ induces a morphism

$$
\partial: H_{\mathrm{A}}^{p, q}(M) \longrightarrow H_{\bar{\partial}}^{p+1, q}(M) .
$$


The third cohomology: We have one more cohomology, i.e., the cohomology of the complex

$$
\mathcal{E}^{p, q}(M) \stackrel{d}{\longrightarrow} \mathcal{E}^{(p, q)+1}(M) \stackrel{\bar{\partial}+\partial}{\longrightarrow} \mathcal{E}^{p+1, q+1}(M),
$$

which will be denoted by $H^{(p, q)+1}(M)$.

\section{Relative Bott-Chern cohomology}

\subsection{Relative cohomology of a complex of sheaves}

A general theory of relative cohomology for complexes of sheaves is developed in [25]. Here we recall some of the relevant materials.

Suppose we have a complex $\left(\mathcal{K}^{\bullet}, d_{\mathcal{K}}\right)$ of sheaves of differential forms on $M$. In fact what we have in mind here are the de Rham and Dolbeault complexes and the one as in (2.1). We denote by $H_{d_{\mathcal{K}}}^{r}(M)$ the $r$-th cohomology of $\left(\mathcal{K} \bullet(M), d_{\mathcal{K}}\right)$.

Čech cohomology of a complex: Let $\mathcal{U}=\left\{U_{0}, U_{1}\right\}$ be an open covering of $M$ and set $U_{01}=U_{0} \cap U_{1}$. We set

$$
\mathcal{K}^{r}(\mathcal{U})=\mathcal{K}^{r}\left(U_{0}\right) \oplus \mathcal{K}^{r}\left(U_{1}\right) \oplus \mathcal{K}^{r-1}\left(U_{01}\right) .
$$

Thus an element $\sigma$ in $\mathcal{K}^{r}(\mathcal{U})$ is given by a triple $\sigma=\left(\sigma_{0}, \sigma_{1}, \sigma_{01}\right)$ with $\sigma_{i} \in \mathcal{K}^{r}\left(U_{i}\right), i=0,1$, and $\sigma_{01} \in \mathcal{K}^{r-1}\left(U_{01}\right)$. We define the differential

$$
D_{\mathcal{K}}=D_{\mathcal{K}}^{r}: \mathcal{K}^{r}(\mathcal{U}) \longrightarrow \mathcal{K}^{r+1}(\mathcal{U}) \quad \text { by } D_{\mathcal{K}} \sigma=\left(d_{\mathcal{K}} \sigma_{0}, d_{\mathcal{K}} \sigma_{1}, \sigma_{1}-\sigma_{0}-d_{\mathcal{K}} \sigma_{01}\right)
$$

Then we see that $D_{\mathcal{K}} \circ D_{\mathcal{K}}=0$.

Definition 3.1 The $r$-th cohomology $H_{D_{\mathcal{K}}}^{r}(\mathcal{U})$ of $\mathcal{K} \bullet$ on $\mathcal{U}$ is the $r$-th cohomology of the complex $\left(\mathcal{K} \bullet(\mathcal{U}), D_{\mathcal{K}}\right)$ :

$$
H_{D_{\mathcal{K}}}^{r}(\mathcal{U})=\operatorname{Ker} D_{\mathcal{K}}^{r} / \operatorname{Im} D_{\mathcal{K}}^{r-1}
$$

If we set $Z_{d_{\mathcal{K}}}^{r}(\mathcal{U})=\operatorname{Ker} D_{\mathcal{K}}^{r}$ and $B_{d_{\mathcal{K}}}^{r}(\mathcal{U})=\operatorname{Im} D_{\mathcal{K}}^{r-1}$, by definition

$$
\begin{aligned}
& Z_{d_{\mathcal{K}}}^{r}(\mathcal{U})=\left\{\sigma \mid d_{\mathcal{K}} \sigma_{0}=d_{\mathcal{K}} \sigma_{1}=0, \quad \sigma_{1}-\sigma_{0}-d_{\mathcal{K}} \sigma_{01}=0\right\}, \\
& B_{d_{\mathcal{K}}}^{r}(\mathcal{U})=\left\{\sigma \mid \exists \tau, \sigma_{0}=d_{\mathcal{K}} \tau_{0}, \sigma_{1}=d_{\mathcal{K}} \tau_{1}, \quad \sigma_{01}=\tau_{1}-\tau_{0}-d_{\mathcal{K}} \tau_{01}\right\},
\end{aligned}
$$

where $\sigma=\left(\sigma_{0}, \sigma_{1}, \sigma_{01}\right) \in \mathcal{K}^{r}(\mathcal{U})$ and $\tau=\left(\tau_{0}, \tau_{1}, \tau_{01}\right) \in \mathcal{K}^{r-1}(\mathcal{U})$. We may somewhat simplify the expression of $B_{d_{\mathcal{K}}}^{r}(\mathcal{U})$, i.e., if we set

$$
B=\left\{\sigma \in \mathcal{K}^{r}(\mathcal{U}) \mid \exists\left(\tau_{0}, \tau_{1}\right), \sigma_{0}=d_{\mathcal{K}} \tau_{0}, \sigma_{1}=d_{\mathcal{K}} \tau_{1}, \quad \sigma_{01}=\tau_{1}-\tau_{0}\right\},
$$

where $\left(\tau_{0}, \tau_{1}\right) \in \mathcal{K}^{r-1}\left(U_{0}\right) \oplus \mathcal{K}^{r-1}\left(U_{1}\right)$, we have :

Lemma $3.3 B_{d_{\mathcal{K}}}^{r}(\mathcal{U})=B$. 
Proof: $\quad$ Clearly we have $B \subset B_{d_{\mathcal{K}}}^{r}(\mathcal{U})$. Suppose $\sigma \in B_{d_{\mathcal{K}}}^{r}(\mathcal{U})$. Then $\sigma_{0}=d_{\mathcal{K}} \tau_{0}, \sigma_{1}=$ $d_{\mathcal{K}} \tau_{1}, \quad \sigma_{01}=\tau_{1}-\tau_{0}-d_{\mathcal{K}} \tau_{01}$. Letting $\left\{\rho_{0}, \rho_{1}\right\}$ be a partition of unity subordinate to $\mathcal{U}$, we set

$$
\tau_{0}^{\prime}=\tau_{0}+d_{\mathcal{K}}\left(\rho_{1} \tau_{01}\right), \quad \tau_{1}^{\prime}=\tau_{1}-d_{\mathcal{K}}\left(\rho_{0} \tau_{01}\right) .
$$

Then $\sigma_{0}=d_{\mathcal{K}} \tau_{0}^{\prime}, \sigma_{1}=d_{\mathcal{K}} \tau_{1}^{\prime}, \quad \sigma_{01}=\tau_{1}^{\prime}-\tau_{0}^{\prime}$.

Theorem 3.4 The map $\mathcal{K}^{r}(M) \longrightarrow \mathcal{K}^{r}(\mathcal{U})$ given by $\omega \mapsto \tilde{\omega}=(\omega, \omega, 0)$ is compatible with the differentials and induces an isomorphism

$$
\alpha: H_{d_{\mathcal{K}}}^{r}(M) \stackrel{\sim}{\longrightarrow} H_{D_{\mathcal{K}}}^{r}(\mathcal{U}) .
$$

Proof: The first part follows from the definitions so that $\alpha$ is well-defined. We show that $\alpha$ is surjective and injective. To see the surjectivity, given $\sigma=\left(\sigma_{0}, \sigma_{1}, \sigma_{01}\right) \in Z_{d_{\mathcal{K}}}^{r}(\mathcal{U})$. We take a partition of unity $\left\{\rho_{0}, \rho_{1}\right\}$ subordinated to $\mathcal{U}$ and set

$$
\omega= \begin{cases}\sigma_{0}+d_{\mathcal{K}}\left(\rho_{1} \sigma_{01}\right) & \text { in } U_{0} \\ \sigma_{1}-d_{\mathcal{K}}\left(\rho_{0} \sigma_{01}\right) & \text { in } U_{1} .\end{cases}
$$

Then we see that it is a cocycle in $\mathcal{K}^{r}(M)$. We claim that $\tilde{\omega}-\sigma=\left(\omega-\sigma_{0}, \omega-\sigma_{1},-\sigma_{01}\right)$ is in $B_{d_{\mathcal{K}}}^{r}(\mathcal{U})$. Indeed, setting $\tau_{0}=\rho_{1} \sigma_{01}, \tau_{1}=-\rho_{0} \sigma_{01}$, we see that

$$
\tilde{\omega}-\sigma=D_{\mathcal{K}}\left(\tau_{0}, \tau_{1}, 0\right),
$$

which shows that $\widetilde{\omega}-\sigma \in B_{d_{\mathcal{K}}}^{r}(\mathcal{U})$ and thus $\alpha$ is surjective.

To see the injectivity of $\alpha$, given $\omega \in \mathcal{K}^{r}(M)$ with $d_{\mathcal{K}} \omega=0$ such that $[\widetilde{\omega}]=0$, we claim that there exist $\theta \in \mathcal{K}^{r-1}(M)$ such that $\omega=d_{\mathcal{K}} \theta$. The condition $[\widetilde{\omega}]=0$ means that there exists $\left(\tau_{0}, \tau_{1}, 0\right)$ such that (cf. Lemma 3.3)

$$
(\omega, \omega, 0)=D_{\mathcal{K}}\left(\tau_{0}, \tau_{1}, 0\right)=\left(d_{\mathcal{K}} \tau_{0}, d_{\mathcal{K}} \tau_{1}, \tau_{1}-\tau_{0}\right)
$$

Thus $\theta=\tau_{0}=\tau_{1}$ is a global form with $\omega=d_{\mathcal{K}} \theta$.

Remark 3.5 The cohomology $H_{D_{\mathcal{K}}}^{r}(\mathcal{U})$ as defined above is the hypercohomology of the complex $\mathcal{K}^{\bullet}$ on $\mathcal{U}$. It can be defined for an arbitrary covering of $M$ and Theorem 3.4 may be proved in the general case, for instance by a spectral sequence argument (cf. [15], [21], $[23],[25])$.

Relative cohomology of a complex : Let $S$ be a closed set in $M$. Setting $U_{0}=M \backslash S$ and $U_{1}$ a neighborhood of $S$, we consider the covering $\mathcal{U}=\left\{U_{0}, U_{1}\right\}$ of $M$. We set

$$
\mathcal{K}^{r}\left(\mathcal{U}, U_{0}\right)=\left\{\sigma \in \mathcal{K}^{r}(\mathcal{U}) \mid \sigma_{0}=0\right\}=\mathcal{K}^{r}\left(U_{1}\right) \oplus \mathcal{K}^{r-1}\left(U_{01}\right) .
$$

Thus an element $\sigma$ in $\mathcal{K}^{r}\left(\mathcal{U}, U_{0}\right)$ is given by a pair $\sigma=\left(\sigma_{1}, \sigma_{01}\right)$ with $\sigma_{1} \in \mathcal{K}^{r}\left(U_{1}\right)$ and $\sigma_{01} \in \mathcal{K}^{r-1}\left(U_{01}\right)$. Clearly $\left(\mathcal{K} \bullet\left(\mathcal{U}, U_{0}\right), D_{\mathcal{K}}\right)$ is a subcomplex of $\left(\mathcal{K} \bullet(\mathcal{U}), D_{\mathcal{K}}\right)$ with $D_{\mathcal{K}}: \mathcal{K}^{r}\left(\mathcal{U}, U_{0}\right) \rightarrow \mathcal{K}^{r+1}\left(\mathcal{U}, U_{0}\right)$ given by $D_{\mathcal{K}} \sigma=\left(d_{\mathcal{K}} \sigma_{1}, \sigma_{1}-d_{\mathcal{K}} \sigma_{01}\right)$. Sometimes $\left(\sigma_{1}, \sigma_{01}\right)$ is denoted by $\left(0, \sigma_{1}, \sigma_{01}\right)$ to remember that it is an element in $\mathcal{K}^{r}(\mathcal{U})$. 
Definition 3.6 The $r$-th relative cohomology $H_{D_{K}}^{r}\left(\mathcal{U}, U_{0}\right)$ of $\mathcal{K}$ is the $r$-th cohomology of the complex $\left(\mathcal{K} \bullet\left(\mathcal{U}, U_{0}\right), D_{\mathcal{K}}\right)$.

Note that, in the relative case, we may not simplify the expression of the coboundaries as in Lemma 3.3.

From the exact sequence of complexes

$$
0 \longrightarrow \mathcal{K}^{\bullet}\left(\mathcal{U}, U_{0}\right) \longrightarrow \mathcal{K}^{\bullet}(\mathcal{U}) \longrightarrow \mathcal{K}^{\bullet}\left(U_{0}\right) \longrightarrow 0,
$$

we have :

Theorem 3.7 There is an exact sequence

$$
\cdots \longrightarrow H_{d_{\mathcal{K}}}^{r-1}\left(U_{0}\right) \stackrel{\delta^{*}}{\longrightarrow} H_{D_{\mathcal{K}}}^{r}\left(\mathcal{U}, U_{0}\right) \stackrel{j^{*}}{\longrightarrow} H_{D_{\mathcal{K}}}^{r}(\mathcal{U}) \stackrel{i^{*}}{\longrightarrow} H_{d_{\mathcal{K}}}^{r}\left(U_{0}\right) \longrightarrow \cdots
$$

where $\delta^{*}[\theta]=[(0,-\theta)], j^{*}\left[\left(\sigma_{1}, \sigma_{01}\right)\right]=\left[\left(0, \sigma_{1}, \sigma_{01}\right)\right]$ and $i^{*}\left[\left(\sigma_{0}, \sigma_{1}, \sigma_{01}\right)\right]=\left[\sigma_{0}\right]$.

By Theorem 3.4 and the five lemma we have:

Corollary 3.8 The cohomology $H_{D_{\mathcal{K}}}^{r}\left(\mathcal{U}, U_{0}\right)$ is determined uniquely up to canonical isomorphisms, independently of the choice of $U_{1}$.

In view of the above, we may denote $H_{D_{\mathcal{K}}}^{r}\left(\mathcal{U}, U_{0}\right)$ by $H_{d_{\mathcal{K}}}^{r}(M, M \backslash S)$. Then we have:

Corollary 3.9 (Excision) For any open set $U$ containing $S$, there is a canonical isomorphism

$$
H_{d_{\mathcal{K}}}^{r}(M, M \backslash S) \simeq H_{d_{\mathcal{K}}}^{r}(U, U \backslash S)
$$

Example 3.10 1. In the case $\mathcal{K}^{\bullet}=\mathcal{E}^{\bullet}$ and $d_{\mathcal{K}}=d$, we write $D_{\mathcal{K}}=D$. The cohomologies defined as above are the Čech-de Rham cohomology $H_{D}^{r}(\mathcal{U})$ and the relative de Rham cohomology $H_{D}^{r}\left(\mathcal{U}, U_{0}\right)$ (cf. [9], [21]). Thus $H_{D}^{r}(\mathcal{U})$ is the cohomology of the complex $(\mathcal{E} \bullet(\mathcal{U}), D)$, where

$$
\mathcal{E}^{r}(\mathcal{U})=\mathcal{E}^{r}\left(U_{0}\right) \oplus \mathcal{E}^{r}\left(U_{1}\right) \oplus \mathcal{E}^{r-1}\left(U_{01}\right)
$$

and $D: \mathcal{E}^{r}(\mathcal{U}) \rightarrow \mathcal{E}^{r+1}(\mathcal{U})$ is given by $D\left(\zeta_{0}, \zeta_{1}, \zeta_{01}\right)=\left(d \zeta_{0}, d \zeta_{1}, \zeta_{1}-\zeta_{0}-d \zeta_{01}\right)$.

By Theorem 3.4, there is a canonical isomorphism

$$
H_{d}^{r}(M) \simeq H_{D}^{r}(\mathcal{U})
$$

The relative de Rham cohomology $H_{D}^{r}\left(\mathcal{U}, U_{0}\right)$ is the cohomology of the subcomplex $\left(\mathcal{E} \bullet\left(\mathcal{U}, U_{0}\right), D\right)$ of $(\mathcal{E} \bullet(\mathcal{U}), D)$, where

$$
\mathcal{E}^{r}\left(\mathcal{U}, U_{0}\right)=\mathcal{E}^{r}\left(U_{1}\right) \oplus \mathcal{E}^{r-1}\left(U_{01}\right) .
$$

2. In the case $\mathcal{K}^{\bullet}=\mathcal{E}^{p} \bullet$ and $d_{\mathcal{K}}=\bar{\partial}$, we write $D_{\mathcal{K}}=\bar{\vartheta}$. The cohomologies as defined above are the Cech-Dolbeault cohomology $H_{\bar{\vartheta}}^{p, q}(\mathcal{U})$ and the relative Dolbeault cohomology $H_{\bar{\vartheta}}^{p, q}\left(\mathcal{U}, U_{0}\right)$ (cf. [23], where the operator $\bar{\vartheta}$ is denoted by $\bar{D}$, and [26]). Thus $H_{\bar{\vartheta}}^{p, q}(\mathcal{U})$ is the cohomology of the complex $\left(\mathcal{E}^{p, \bullet}(\mathcal{U}), \bar{\vartheta}\right)$, where

$$
\mathcal{E}^{p, q}(\mathcal{U})=\mathcal{E}^{p, q}\left(U_{0}\right) \oplus \mathcal{E}^{p, q}\left(U_{1}\right) \oplus \mathcal{E}^{p, q-1}\left(U_{01}\right)
$$


and $\bar{\vartheta}: \mathcal{E}^{p, q}(\mathcal{U}) \rightarrow \mathcal{E}^{p, q+1}(\mathcal{U})$ is given by $\bar{\vartheta}\left(\zeta_{0}, \zeta_{1}, \zeta_{01}\right)=\left(\bar{\partial} \zeta_{0}, \bar{\partial} \zeta_{1}, \zeta_{1}-\zeta_{0}-\bar{\partial} \zeta_{01}\right)$.

By Theorem 3.4, there is a canonical isomorphism

$$
H_{\bar{\partial}}^{p, q}(M) \simeq H_{\bar{\vartheta}}^{p, q}(\mathcal{U})
$$

The relative Dolbeault cohomology $H_{D}^{p, q}\left(\mathcal{U}, U_{0}\right)$ is the cohomology of the subcomplex $\left(\mathcal{E}^{p, \bullet}\left(\mathcal{U}, U_{0}\right), \bar{\vartheta}\right)$ of $\left(\mathcal{E}^{p, \bullet}(\mathcal{U}), \bar{\vartheta}\right)$, where

$$
\mathcal{E}^{p, q}\left(\mathcal{U}, U_{0}\right)=\mathcal{E}^{p, q}\left(U_{1}\right) \oplus \mathcal{E}^{p, q-1}\left(U_{01}\right) .
$$

In the sequel we examine each cohomology arising from (2.1). For this we set

$$
\begin{aligned}
\mathcal{E}_{\mathrm{BC}}^{p, q}(\mathcal{U}) & =\mathcal{E}^{p, q}\left(U_{0}\right) \oplus \mathcal{E}^{p, q}\left(U_{1}\right) \oplus \mathcal{E}^{p-1, q-1}\left(U_{01}\right), \\
\mathcal{E}_{\mathrm{A}}^{p, q}(\mathcal{U}) & =\mathcal{E}^{p, q}\left(U_{0}\right) \oplus \mathcal{E}^{p, q}\left(U_{1}\right) \oplus \mathcal{E}^{(p-1, q-1)+1}\left(U_{01}\right), \\
\mathcal{E}^{(p, q)+1}(\mathcal{U}) & =\mathcal{E}^{(p, q)+1}\left(U_{0}\right) \oplus \mathcal{E}^{(p, q)+1}\left(U_{1}\right) \oplus \mathcal{E}^{p, q}\left(U_{01}\right)
\end{aligned}
$$

and

$$
\begin{aligned}
\mathcal{E}_{\mathrm{BC}}^{p, q}\left(\mathcal{U}, U_{0}\right) & =\mathcal{E}^{p, q}\left(U_{1}\right) \oplus \mathcal{E}^{p-1, q-1}\left(U_{01}\right), \\
\mathcal{E}_{\mathrm{A}}^{p, q}\left(\mathcal{U}, U_{0}\right) & =\mathcal{E}^{p, q}\left(U_{1}\right) \oplus \mathcal{E}^{(p-1, q-1)+1}\left(U_{01}\right), \\
\mathcal{E}^{(p, q)+1}\left(\mathcal{U}, U_{0}\right) & =\mathcal{E}^{(p, q)+1}\left(U_{1}\right) \oplus \mathcal{E}^{p, q}\left(U_{01}\right) .
\end{aligned}
$$

\subsection{Relative Bott-Chern cohomology}

The relevant part of the complex is

$$
\mathcal{E}^{(p-2, q-2)+1} \stackrel{\bar{\partial}+\partial}{\longrightarrow} \mathcal{E}^{p-1, q-1} \stackrel{\bar{\partial} \partial}{\longrightarrow} \mathcal{E}^{p, q} \stackrel{d}{\longrightarrow} \mathcal{E}^{(p, q)+1} .
$$

Čech-Bott-Chern cohomology : The group of Čech-Bott-Chern cochains of type $(p, q)$ is $\mathcal{E}_{\mathrm{BC}}^{p, q}(\mathcal{U})$. Thus an element $\sigma$ in $\mathcal{E}_{\mathrm{BC}}^{p, q}(\mathcal{U})$ is given by a triple $\sigma=\left(\sigma_{0}, \sigma_{1}, \sigma_{01}\right)$. The groups of cocycles and coboundaries are given by (cf. (3.2))

$$
\begin{aligned}
& Z_{\mathrm{BC}}^{p, q}(\mathcal{U})=\left\{\sigma \mid d \sigma_{0}=d \sigma_{1}=0, \quad \sigma_{1}-\sigma_{0}-\bar{\partial} \partial \sigma_{01}=0\right\}, \\
& B_{\mathrm{BC}}^{p, q}(\mathcal{U})=\left\{\sigma \mid \exists \tau, \sigma_{0}=\bar{\partial} \partial \tau_{0}, \quad \sigma_{1}=\bar{\partial} \partial \tau_{1}, \quad \sigma_{01}=\tau_{1}-\tau_{0}-\bar{\partial} \tau_{01}^{(1)}-\partial \tau_{01}^{(2)}\right\},
\end{aligned}
$$

where $\sigma \in \mathcal{E}_{\mathrm{BC}}^{p, q}(\mathcal{U})$ and $\tau=\left(\tau_{0}, \tau_{1}, \tau_{01}^{(1)}, \tau_{01}^{(2)}\right) \in \mathcal{E}_{\mathrm{A}}^{p-1, q-1}(\mathcal{U})$.

Definition 3.16 The Čech-Bott-Chern cohomology of type $(p, q)$ of $\mathcal{U}$ is defined by

$$
H_{\mathrm{BC}}^{p, q}(\mathcal{U})=Z_{\mathrm{BC}}^{p, q}(\mathcal{U}) / B_{\mathrm{BC}}^{p, q}(\mathcal{U}) .
$$

By Theorem 3.4, the map $\mathcal{E}^{p, q}(M) \rightarrow \mathcal{E}_{\mathrm{BC}}^{p, q}(\mathcal{U})$ given by $\omega \mapsto(\omega, \omega, 0)$ induces an isomorphism

$$
H_{\mathrm{BC}}^{p, q}(M) \stackrel{\sim}{\longrightarrow} H_{\mathrm{BC}}^{p, q}(\mathcal{U}) .
$$

The inverse assigns to the class of $\sigma=\left(\sigma_{0}, \sigma_{1}, \sigma_{01}\right)$ the class of the global form

$$
\omega=\rho_{0} \sigma_{0}+\rho_{1} \sigma_{1}+\partial \rho_{0} \wedge \bar{\partial} \sigma_{01}-\bar{\partial} \rho_{0} \wedge \partial \sigma_{01}-\bar{\partial} \partial \rho_{0} \wedge \sigma_{01}
$$

where $\left(\rho_{0}, \rho_{1}\right)$ is a partition of unity subordinate to $\mathcal{U}$.

By Lemma 3.3, we may simplify the expression of the coboundary group, i.e., we may drop $\tau_{01}^{(1)}$ and $\tau_{01}^{(2)}$ in (3.15). 
Relative Bott-Chern cohomology: The group of relative Bott-Chern cochains is $\mathcal{E}_{\mathrm{BC}}^{p, q}\left(\mathcal{U}, U_{0}\right)$. The relative cocycle and coboundary groups are given by

$$
\begin{aligned}
& Z_{\mathrm{BC}}^{p, q}\left(\mathcal{U}, U_{0}\right)=\left\{\sigma \mid d \sigma_{1}=0, \sigma_{1}-\bar{\partial} \partial \sigma_{01}=0\right\}, \\
& B_{\mathrm{BC}}^{p, q}\left(\mathcal{U}, U_{0}\right)=\left\{\sigma \mid \exists \tau, \sigma_{1}=\bar{\partial} \partial \tau_{1}, \quad \sigma_{01}=\tau_{1}-\bar{\partial} \tau_{01}^{(1)}-\partial \tau_{01}^{(2)}\right\},
\end{aligned}
$$

where $\sigma \in \mathcal{E}_{\mathrm{BC}}^{p, q}\left(\mathcal{U}, U_{0}\right)$ and $\tau=\left(\tau_{1}, \tau_{01}^{(1)}, \tau_{01}^{(2)}\right) \in \mathcal{E}_{\mathrm{A}}^{p-1, q-1}\left(\mathcal{U}, U_{0}\right)$.

Definition 3.18 The relative Bott-Chern cohomology is defined by

$$
H_{\mathrm{BC}}^{p, q}\left(\mathcal{U}, U_{0}\right)=Z_{\mathrm{BC}}^{p, q}\left(\mathcal{U}, U_{0}\right) / B_{\mathrm{BC}}^{p, q}\left(\mathcal{U}, U_{0}\right) .
$$

\subsection{Relative Aeppli cohomology}

The relevant part of the complex is

$$
\mathcal{E}^{p-1, q-1} \stackrel{d}{\longrightarrow} \mathcal{E}^{(p-1, q-1)+1} \stackrel{\bar{\partial}+\partial}{\longrightarrow} \mathcal{E}^{p, q} \stackrel{\bar{\partial} \partial}{\longrightarrow} \mathcal{E}^{p+1, q+1} .
$$

Čech-Aeppli cohomology: Let $\mathcal{U}=\left\{U_{0}, U_{1}\right\}$ be an open covering of $M$ as before. The group of $\check{C}$ ech-Aeppli cochains of type $(p, q)$ is $\mathcal{E}_{\mathrm{A}}^{p, q}(\mathcal{U})$. Thus an element $\xi$ in $\mathcal{E}_{\mathrm{A}}^{p, q}(\mathcal{U})$ is given by a quadruple $\left(\xi_{0}, \xi_{1}, \xi_{01}^{(1)}, \xi_{01}^{(2)}\right)$. The cocycle and coboundary groups of type $(p, q)$ are given by

$$
\begin{aligned}
& Z_{\mathrm{A}}^{p, q}(\mathcal{U})=\left\{\xi \mid \bar{\partial} \partial \xi_{0}=\bar{\partial} \partial \xi_{1}=0, \quad \xi_{1}-\xi_{0}-\bar{\partial} \xi_{01}^{(1)}-\partial \xi_{01}^{(2)}=0\right\}, \\
& B_{\mathrm{A}}^{p, q}(\mathcal{U})=\left\{\xi \mid \exists \eta, \quad \xi_{i}=\bar{\partial} \eta_{i}^{(1)}+\partial \eta_{i}^{(2)}, \quad \xi_{01}^{(1)}=\eta_{1}^{(1)}-\eta_{0}^{(1)}-\partial \eta_{01}, \quad \xi_{01}^{(2)}=\eta_{1}^{(2)}-\eta_{0}^{(2)}-\bar{\partial} \eta_{01}\right\},
\end{aligned}
$$

where $\xi \in \mathcal{E}_{\mathrm{A}}^{p, q}(\mathcal{U})$ and $\eta=\left(\eta_{0}^{(1)}, \eta_{0}^{(2)}, \eta_{1}^{(1)}, \eta_{1}^{(2)}, \eta_{01}\right) \in \mathcal{E}^{(p-1, q-1)+1}(\mathcal{U})$, i.e., $\eta_{i}^{(1)} \in \mathcal{E}^{p, q-1}\left(U_{i}\right)$, $\eta_{i}^{(2)} \in \mathcal{E}^{p-1, q}\left(U_{i}\right), i=0,1$, and $\eta_{01} \in \mathcal{E}^{p-1, q-1}\left(U_{01}\right)$.

Definition 3.20 The Čech-Aeppli cohomology of type $(p, q)$ of $\mathcal{U}$ is defined by

$$
H_{\mathrm{A}}^{p, q}(\mathcal{U})=Z_{\mathrm{A}}^{p, q}(\mathcal{U}) / B_{\mathrm{A}}^{p, q}(\mathcal{U})
$$

By Theorem 3.4, the map $\mathcal{E}^{p, q}(M) \rightarrow \mathcal{E}_{\mathrm{A}}^{p, q}(\mathcal{U})$ given by $\omega \mapsto(\omega, \omega, 0,0)$ induces an isomorphism

$$
H_{\mathrm{A}}^{p, q}(M) \stackrel{\sim}{\longrightarrow} H_{\mathrm{A}}^{p, q}(\mathcal{U}) .
$$

The inverse is given by assigning to the class of $\sigma$ the class of the global form

$$
\omega=\rho_{0} \xi_{0}+\rho_{1} \xi_{1}-\partial \rho_{0} \wedge \xi_{01}^{(1)}-\bar{\partial} \rho_{0} \wedge \xi_{01}^{(2)} .
$$

By Lemma 3.3, we may simplify the expression of the coboundary group, i.e., we may set $\eta_{01}=0$ in (3.19).

We may also relax the coboundary condition. Thus we set

$$
B^{\prime}=\left\{\xi \mid \xi_{i}=\bar{\partial} \eta_{i}^{(1)}+\partial \eta_{i}^{(2)}, \xi_{01}^{(k)}=\eta_{1}^{(k)}-\eta_{0}^{(k)}-\bar{\partial} \eta_{01}^{(k 1)}-\partial \eta_{01}^{(k 2)}, i=0,1, k=1,2\right\},
$$

where $\xi \in \mathcal{E}_{\mathrm{A}}^{p, q}(\mathcal{U}), \eta_{i}^{(1)} \in \mathcal{E}^{p, q-1}\left(U_{i}\right), \eta_{i}^{(2)} \in \mathcal{E}^{p-1, q}\left(U_{i}\right), i=0,1, \eta_{01}^{(11)} \in \mathcal{E}^{p, q-2}\left(U_{01}\right)$, $\eta_{01}^{(12)}=\eta_{01}^{(21)} \in \mathcal{E}^{p-1, q-1}\left(U_{01}\right), \eta_{01}^{(22)} \in \mathcal{E}^{p-2, q}\left(U_{01}\right)$. 
Lemma 3.22 We have $B_{A}^{p, q}(\mathcal{U})=B^{\prime}$.

Proof: $\quad$ Clearly $B_{A}^{p, q}(\mathcal{U}) \subset B^{\prime}$. Take an element $\xi \in B^{\prime}$. Denoting by $\left\{\rho_{0}, \rho_{1}\right\}$ a partition of unity subordinate to $\mathcal{U}$ we set

$$
\chi_{0}^{(k)}=\eta_{0}^{(k)}+\bar{\partial}\left(\rho_{1} \eta_{01}^{(k 1)}\right)+\partial\left(\rho_{1} \eta_{01}^{(k 2)}\right), \quad \chi_{1}^{(k)}=\eta_{1}^{(k)}-\bar{\partial}\left(\rho_{0} \eta_{01}^{(k 1)}\right)-\partial\left(\rho_{0} \eta_{01}^{(k 2)}\right) .
$$

Then $\xi_{i}=\bar{\partial} \chi_{i}^{(1)}+\partial \chi_{i}^{(2)}, \xi_{01}^{(k)}=\chi_{1}^{(k)}-\chi_{0}^{(k)}, i=0,1, k=1,2$.

The following is proved by applying Lemma 3.3 for the Čech-de Rham coboundaries or by applying Lemma 3.22 :

Proposition 3.23 The morphism $\mathcal{E}_{\mathrm{A}}^{n, n}(\mathcal{U}) \rightarrow \mathcal{E}^{2 n}(\mathcal{U})$ given by

$$
\left(\xi_{0}, \xi_{1}, \xi_{01}^{(1)}, \xi_{01}^{(2)}\right) \mapsto\left(\xi_{0}, \xi_{1}, \xi_{01}^{(1)}+\xi_{01}^{(2)}\right)
$$

induces an isomorphism

$$
H_{\mathrm{A}}^{n, n}(\mathcal{U}) \stackrel{\sim}{\longrightarrow} H_{D}^{2 n}(\mathcal{U})
$$

The above isomorphism is compatible with the identity $H_{A}^{n, n}(M)=H_{d}^{2 n}(M)$ via the isomorphisms of Theorem 3.4 for the Aeppli and de Rham cases.

Relative Aeppli cohomology : Let $S$ be a closed set in $M$. Setting $U_{0}=M \backslash S$ and $U_{1}$ a neighborhood of $S$, we consider the covering $\mathcal{U}=\left\{U_{0}, U_{1}\right\}$ of $M$. The group of relative Aeppli cochains is $\mathcal{E}_{\mathrm{A}}^{p, q}\left(\mathcal{U}, U_{0}\right)$. The relative cocycle and coboundary groups are given by

$$
\begin{aligned}
& Z_{\mathrm{A}}^{p, q}\left(\mathcal{U}, U_{0}\right)=\left\{\xi \mid \bar{\partial} \partial \xi_{1}=0, \quad \xi_{1}-\bar{\partial} \xi_{01}^{(1)}-\partial \xi_{01}^{(2)}=0\right\}, \\
& B_{\mathrm{A}}^{p, q}\left(\mathcal{U}, U_{0}\right)=\left\{\xi \mid \exists \eta, \quad \xi_{1}=\bar{\partial} \eta_{1}^{(1)}+\partial \eta_{1}^{(2)}, \xi_{01}^{(1)}=\eta_{1}^{(1)}-\partial \eta_{01}, \quad \xi_{01}^{(2)}=\eta_{1}^{(2)}-\bar{\partial} \eta_{01}\right\},
\end{aligned}
$$

where $\xi \in \mathcal{E}_{\mathrm{A}}^{p, q}\left(\mathcal{U}, U_{0}\right)$ and $\eta=\left(\eta_{1}^{(1)}, \eta_{1}^{(2)}, \eta_{01}\right) \in \mathcal{E}^{(p-1, q-1)+1}\left(\mathcal{U}, U_{0}\right)$.

Definition 3.24 The relative Aeppli cohomology is defined by

$$
H_{\mathrm{A}}^{p, q}\left(\mathcal{U}, U_{0}\right)=Z_{\mathrm{A}}^{p, q}\left(\mathcal{U}, U_{0}\right) / B_{\mathrm{A}}^{p, q}\left(\mathcal{U}, U_{0}\right)
$$

In the relative case, Lemma 3.3 or 3.22 does not apply and we do not have an isomorphism as in Proposition 3.23. However we have:

Proposition 3.25 The morphism $\mathcal{E}_{\mathrm{A}}^{n, n}\left(\mathcal{U}, U_{0}\right) \rightarrow \mathcal{E}^{2 n}\left(\mathcal{U}, U_{0}\right)$ given by

$$
\left(\xi_{1}, \xi_{01}^{(1)}, \xi_{01}^{(2)}\right) \mapsto\left(\xi_{1}, \xi_{01}^{(1)}+\xi_{01}^{(2)}\right)
$$

induces an epimorphism

$$
H_{\mathrm{A}}^{n, n}\left(\mathcal{U}, U_{0}\right) \longrightarrow H_{D}^{2 n}\left(\mathcal{U}, U_{0}\right)
$$




\subsection{An exact sequence}

Considering

$$
\mathcal{E}^{p-1, q-1} \stackrel{\bar{\partial} \partial}{\longrightarrow} \mathcal{E}^{p, q} \stackrel{d}{\longrightarrow} \mathcal{E}^{(p, q)+1} \stackrel{\bar{\partial}+\partial}{\longrightarrow} \mathcal{E}^{p+1, q+1},
$$

we may also define $H^{(p, q)+1}(\mathcal{U})$ and $H^{(p, q)+1}\left(\mathcal{U}, U_{0}\right)$ using the cochains $\mathcal{E}^{(p, q)+1}(\mathcal{U})$ and $\mathcal{E}^{(p, q)+1}\left(\mathcal{U}, U_{0}\right)$ (cf. (3.13) and (3.14)).

By focusing on the Bott-Chern cohomology in Theorm 3.7, we have:

Theorem 3.26 There is an exact sequence

$$
\begin{aligned}
& \cdots \longrightarrow H_{\mathrm{A}}^{p-1, q-1}(\mathcal{U}) \longrightarrow H_{\mathrm{A}}^{p-1, q-1}\left(U_{0}\right) \stackrel{\delta^{*}}{\longrightarrow} H_{\mathrm{BC}}^{p, q}\left(\mathcal{U}, U_{0}\right) \stackrel{j^{*}}{\longrightarrow} H_{\mathrm{BC}}^{p, q}(\mathcal{U}) \stackrel{i^{*}}{\longrightarrow} H_{\mathrm{BC}}^{p, q}\left(U_{0}\right) \\
& \longrightarrow H^{(p, q)+1}\left(\mathcal{U}, U_{0}\right) \longrightarrow H^{(p, q)+1}(\mathcal{U}) \longrightarrow H^{(p, q)+1}\left(U_{0}\right) \longrightarrow H_{\mathrm{A}}^{p+1, q+1}\left(\mathcal{U}, U_{0}\right) \longrightarrow \cdots
\end{aligned}
$$

Now we discuss the relation with the Dolbeault case (cf. Example 3.10.2). The following two propositions follow directly from the definition:

Proposition 3.27 1. The map $\mathcal{E}_{\mathrm{BC}}^{p, q}(\mathcal{U}) \rightarrow \mathcal{E}^{p, q}(\mathcal{U})$ given by $\left(\sigma_{0}, \sigma_{1}, \sigma_{01}\right) \mapsto\left(\sigma_{0}, \sigma_{1}, \partial \sigma_{01}\right)$ induces a morphism

$$
H_{\mathrm{BC}}^{p, q}(\mathcal{U}) \longrightarrow H_{\bar{\vartheta}}^{p, q}(\mathcal{U})
$$

which is compatible with the first morphism in (2.2) via the isomorphisms (3.17) and (3.12).

2. The map $\mathcal{E}_{\mathrm{BC}}^{p, q}\left(\mathcal{U}, U_{0}\right) \rightarrow \mathcal{E}^{p, q}\left(\mathcal{U}, U_{0}\right)$ given by $\left(\sigma_{1}, \sigma_{01}\right) \mapsto\left(\sigma_{1}, \partial \sigma_{01}\right)$ induces a morphism

$$
H_{\mathrm{BC}}^{p, q}\left(\mathcal{U}, U_{0}\right) \longrightarrow H_{\bar{\vartheta}}^{p, q}\left(\mathcal{U}, U_{0}\right) \text {. }
$$

Proposition 3.28 1. The map $\mathcal{E}_{\mathrm{A}}^{p, q}(\mathcal{U}) \rightarrow \mathcal{E}^{p+1, q}(\mathcal{U})$ given by

$$
\left(\xi_{0}, \xi_{1}, \xi_{01}^{(1)}, \xi_{01}^{(2)}\right) \mapsto\left(\partial \xi_{0}, \partial \xi_{1},-\partial \xi_{01}^{(1)}\right)
$$

induces a morphism

$$
\partial: H_{\mathrm{A}}^{p, q}(\mathcal{U}) \longrightarrow H_{\bar{\vartheta}}^{p+1, q}(\mathcal{U}),
$$

which is compatible with the morphism (2.4) via the isomorphisms (3.21) and (3.12).

2. The map $\mathcal{E}_{\mathrm{A}}^{p, q}\left(\mathcal{U}, U_{0}\right) \rightarrow \mathcal{E}^{p+1, q}\left(\mathcal{U}, U_{0}\right)$ given by

$$
\left(\xi_{1}, \xi_{01}^{(1)}, \xi_{01}^{(2)}\right) \mapsto\left(\partial \xi_{1},-\partial \xi_{01}^{(1)}\right)
$$

induces a morphism

$$
\partial: H_{\mathrm{A}}^{p, q}\left(\mathcal{U}, U_{0}\right) \longrightarrow H_{\bar{\vartheta}}^{p+1, q}\left(\mathcal{U}, U_{0}\right)
$$

Theorem 3.29 We have the following commutative diagram with exact rows:

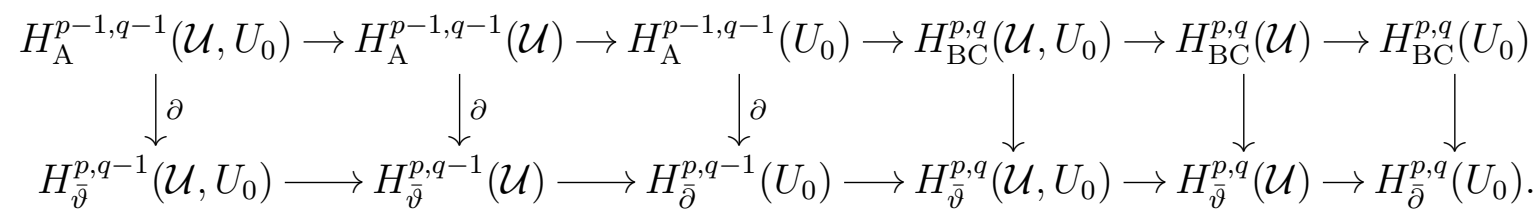

In the above, the first row is a part of the sequence in Theorem 3.26 and the second row is the one in Theorem 3.7 for the Dolbeault complex (cf. Example 3.10.2). 


\section{Cup product, integration and duality}

Let $M$ be a complex manifold of dimension $n$.

\subsection{Cup product}

Recall that the exterior product induces a bilinear map (cf. [20])

$$
H_{\mathrm{BC}}^{p, q}(M) \times H_{\mathrm{A}}^{r, s}(M) \stackrel{\wedge}{\longrightarrow} H_{\mathrm{A}}^{p+r, q+s}(M) .
$$

Now we try to find a product in Čech-Bott-Chern and Čech-Aeppli cohomologies that corresponds to the above. We define a cup product

$$
\mathcal{E}_{\mathrm{BC}}^{p, q}(\mathcal{U}) \times \mathcal{E}_{\mathrm{A}}^{r, s}(\mathcal{U}) \stackrel{\smile}{\longrightarrow} \mathcal{E}_{\mathrm{A}}^{p+r, q+s}(\mathcal{U})
$$

by assigning to $(\sigma, \xi), \sigma=\left(\sigma_{0}, \sigma_{1}, \sigma_{01}\right), \xi=\left(\xi_{0}, \xi_{1}, \xi_{01}^{(1)}, \xi_{01}^{(2)}\right)$, the cochain $\sigma \smile \xi$ given by

$$
\begin{aligned}
(\sigma \smile \xi)_{i} & =\sigma_{i} \wedge \xi_{i}, \quad i=0,1, \\
(\sigma \smile \xi)_{01}^{(1)} & =(-1)^{p+q} \sigma_{0} \wedge \xi_{01}^{(1)}+\partial \sigma_{01} \wedge \xi_{1}-\frac{1}{2} \partial\left(\sigma_{01} \wedge \xi_{1}\right), \\
(\sigma \smile \xi)_{01}^{(2)} & =(-1)^{p+q} \sigma_{0} \wedge \xi_{01}^{(2)}-\bar{\partial} \sigma_{01} \wedge \xi_{1}+\frac{1}{2} \bar{\partial}\left(\sigma_{01} \wedge \xi_{1}\right) .
\end{aligned}
$$

Proposition 4.3 The above cup product induces a bilinear map

$$
H_{\mathrm{BC}}^{p, q}(\mathcal{U}) \times H_{\mathrm{A}}^{r, s}(\mathcal{U}) \stackrel{\smile}{\longrightarrow} H_{\mathrm{A}}^{p+r, q+s}(\mathcal{U}) .
$$

Proof: We need to show that

(1) If $\sigma \in Z_{\mathrm{BC}}^{p, q}(\mathcal{U})$ and $\xi \in Z_{\mathrm{A}}^{r, s}(\mathcal{U})$, then $\sigma \smile \xi \in Z_{\mathrm{A}}^{p+r, q+s}(\mathcal{U})$.

(2) If $\sigma \in B_{\mathrm{BC}}^{p, q}(\mathcal{U})$ and $\xi \in Z_{\mathrm{A}}^{r, s}(\mathcal{U})$, then $\sigma \smile \xi \in B_{\mathrm{A}}^{p+r, q+s}(\mathcal{U})$.

(3) If $\sigma \in Z_{\mathrm{BC}}^{p, q}(\mathcal{U})$ and $\xi \in B_{\mathrm{A}}^{r, s}(\mathcal{U})$, then $\sigma \smile \xi \in B_{\mathrm{A}}^{p+r, q+s}(\mathcal{U})$.

First, we note that, for a $(p, q)$-form $\omega$ and an $(r, s)$-form $\theta$,

$$
\bar{\partial} \partial(\omega \wedge \theta)=\bar{\partial} \partial \omega \wedge \theta+(-1)^{p+q+1} \partial \omega \wedge \bar{\partial} \theta+(-1)^{p+q} \bar{\partial} \omega \wedge \partial \theta+\omega \wedge \bar{\partial} \partial \theta .
$$

The statement (1) follows from direct computations using (4.4).

To prove $(2)$, take $\sigma \in B_{\mathrm{BC}}^{p, q}(\mathcal{U})$ and $\xi \in Z_{\mathrm{A}}^{r, s}(\mathcal{U})$. Thus $\sigma_{i}=\bar{\partial} \partial \tau_{i}, i=0,1$, and $\sigma_{01}=\tau_{1}-\tau_{0}$ (cf. Lemma 3.3). Also $\bar{\partial} \partial \xi_{i}=0, i=0,1$, and $\xi_{1}-\xi_{0}-\bar{\partial} \xi_{01}^{(1)}-\partial \xi_{01}^{(2)}=0$. Then we may write

$$
(\sigma \smile \xi)_{i}=\bar{\partial} \alpha_{i}^{(1)}+\partial \alpha_{i}^{(2)},
$$

where

$$
\alpha_{i}^{(1)}=-(-1)^{p+q} \tau_{i} \wedge \partial \xi_{i}+\frac{1}{2} \partial\left(\tau_{i} \wedge \xi_{i}\right), \quad \alpha_{i}^{(2)}=(-1)^{p+q} \tau_{i} \wedge \bar{\partial} \xi_{i}-\frac{1}{2} \bar{\partial}\left(\tau_{i} \wedge \xi_{i}\right) .
$$


We compute

$$
(\sigma \smile \xi)_{01}^{(1)}=\alpha_{1}^{(1)}-\alpha_{0}^{(1)}-\bar{\partial} \alpha_{01}^{(11)}-\partial \alpha_{01}^{(12)}, \quad(\sigma \smile \xi)_{01}^{(2)}=\alpha_{1}^{(2)}-\alpha_{0}^{(2)}-\bar{\partial} \alpha_{01}^{(21)}-\partial \alpha_{01}^{(22)},
$$

where

$$
\begin{aligned}
& \alpha_{01}^{(11)}=-(-1)^{p+q} \partial \tau_{0} \wedge \xi_{01}^{(1)}, \quad \alpha_{01}^{(12)}=\alpha_{01}^{(21)}=-\frac{1}{2} \tau_{0} \wedge\left(\bar{\partial} \xi_{01}^{(1)}-\partial \xi_{01}^{(2)}\right), \\
& \alpha_{01}^{(22)}=(-1)^{p+q} \bar{\partial} \tau_{0} \wedge \xi_{01}^{(2)} .
\end{aligned}
$$

Thus $\sigma \smile \xi \in B_{\mathrm{A}}^{p+r, q+s}(\mathcal{U})$ (cf. Lemma 3.22).

To prove $(3)$, take $\sigma \in Z_{\mathrm{BC}}^{p, q}(\mathcal{U})$ and $\xi \in B_{\mathrm{A}}^{r, s}(\mathcal{U})$. Thus $d \sigma_{i}=0, i=0,1, \sigma_{1}-\sigma_{0}-$ $\bar{\partial} \partial \sigma_{01}=0$ and $\xi_{i}=\bar{\partial} \eta_{i}^{(1)}+\partial \eta_{i}^{(2)}, \xi_{01}^{(k)}=\eta_{1}^{(k)}-\eta_{0}^{(k)}, i=0,1, k=1,2$, (cf. Lemma 3.3). Then we may write

$$
(\sigma \smile \xi)_{i}=\bar{\partial} \beta_{i}^{(1)}+\partial \beta_{i}^{(2)}, \quad \beta_{i}^{(k)}=(-1)^{p+q} \sigma_{i} \wedge \eta_{i}^{(k)} .
$$

We compute

$$
(\sigma \smile \xi)_{01}^{(1)}=\beta_{1}^{(1)}-\beta_{0}^{(1)}-\bar{\partial} \beta_{01}^{(11)}-\partial \beta_{01}^{(12)}, \quad(\sigma \smile \xi)_{01}^{(2)}=\beta_{1}^{(2)}-\beta_{0}^{(2)}-\bar{\partial} \beta_{01}^{(21)}-\partial \beta_{01}^{(22)},
$$

where

$$
\begin{aligned}
& \beta_{01}^{(11)}=(-1)^{p+q} \partial \sigma_{01} \wedge \eta_{1}^{(1)}, \quad \beta_{01}^{(12)}=\beta_{01}^{(21)}=\frac{1}{2} \sigma_{01} \wedge\left(\bar{\partial} \eta_{1}^{(1)}-\partial \eta_{1}^{(2)}\right), \\
& \beta_{01}^{(22)}=-(-1)^{p+q} \bar{\partial} \sigma_{01} \wedge \eta_{1}^{(2)} .
\end{aligned}
$$

Thus $\sigma \smile \xi \in B_{\mathrm{A}}^{p+r, q+s}(\mathcal{U})$.

Note that the cup product in Proposition 4.3 is compatible with (4.1) via the isomorphisms (3.17) and (3.21).

Remark 4.5 If we do not use Lemma 3.3 and write $\sigma_{01}=\tau_{1}-\tau_{0}-\bar{\partial} \tau_{01}^{(1)}-\partial \tau_{01}^{(2)}$ in the step (2) above, the terms $(\sigma \smile \xi)_{i}, i=0,1$, remain the same and we have

$$
\begin{aligned}
& (\sigma \smile \xi)_{01}^{(1)}=\alpha_{1}^{(1)}-\alpha_{0}^{(1)}-\bar{\partial}\left(\alpha_{01}^{(11)}+\gamma_{01}^{(11)}\right)-\partial\left(\alpha_{01}^{(12)}+\gamma_{01}^{(12)}\right), \\
& (\sigma \smile \xi)_{01}^{(2)}=\alpha_{1}^{(2)}-\alpha_{0}^{(2)}-\bar{\partial}\left(\alpha_{01}^{(21)}+\gamma_{01}^{(21)}\right)-\partial\left(\alpha_{01}^{(22)}+\gamma_{01}^{(22)}\right),
\end{aligned}
$$

where $\alpha_{01}^{(k l)}, k, l=1,2$, are as before and

$$
\begin{aligned}
& \gamma_{01}^{(11)}=-(-1)^{p+q} \tau_{01}^{(1)} \wedge \partial \xi_{1}, \quad \gamma_{01}^{(12)}=\gamma_{01}^{(21)}=\frac{1}{2}\left(\bar{\partial} \tau_{01}^{(1)}-\partial \tau_{01}^{(2)}\right) \wedge \xi_{1}, \\
& \gamma_{01}^{(22)}=(-1)^{p+q} \tau_{01}^{(2)} \wedge \bar{\partial} \xi_{1} .
\end{aligned}
$$

Thus $\sigma \smile \xi$ is still in $B_{\mathrm{A}}^{p+r, q+s}(\mathcal{U})$ (cf. Lemma 3.22). This is necessary when we consider the relative case (cf. the proof of Proposition 4.9 below). 
Now we consider the relative case. Thus let $S$ be a closed set in $M$. Let $U_{0}=M \backslash S$ and $U_{1}$ a neighborhood of $S$ and consider the covering $\mathcal{U}=\left\{U_{0}, U_{1}\right\}$ as before. By setting $\sigma_{0}=0$ in (4.2), we see that it induces a cup product

$$
\mathcal{E}_{\mathrm{BC}}^{p, q}\left(\mathcal{U}, U_{0}\right) \times \mathcal{E}^{r, s}\left(U_{1}\right) \stackrel{\smile}{\longrightarrow} \mathcal{E}_{\mathrm{A}}^{p+r, q+s}\left(\mathcal{U}, U_{0}\right)
$$

given by

$$
(\sigma, \xi) \mapsto\left(\sigma_{1} \wedge \xi_{1}, \partial \sigma_{01} \wedge \xi_{1}-\frac{1}{2} \partial\left(\sigma_{01} \wedge \xi_{1}\right),-\bar{\partial} \sigma_{01} \wedge \xi_{1}+\frac{1}{2} \bar{\partial}\left(\sigma_{01} \wedge \xi_{1}\right)\right) .
$$

It does not induce the cup product on the corresponding cohomologies, since Lemma 3.22 does not apply in the relative case. However, for our purpose it suffices to consider the case $p+r=q+s=n$ and in this case, we can make the receiving cohomology the relative de Rham cohomology (cf. Example 3.10.1). Namely, if we consider the composition of $(4.7)$ and $\mathcal{E}_{A}^{n, n}\left(\mathcal{U}, U_{0}\right) \rightarrow \mathcal{E}^{2 n}\left(\mathcal{U}, U_{0}\right)$ given by $\left(\xi_{1}, \xi_{01}^{(1)}, \xi_{01}^{(2)}\right) \mapsto\left(\xi_{1}, \xi_{01}^{(1)}+\xi_{01}^{(2)}\right)$ (cf. Proposition 3.25), we have a bilinear map

$$
\mathcal{E}_{\mathrm{BC}}^{p, q}\left(\mathcal{U}, U_{0}\right) \times \mathcal{E}^{n-p, n-q}\left(U_{1}\right) \stackrel{\cdot}{\longrightarrow} \mathcal{E}^{2 n}\left(\mathcal{U}, U_{0}\right)
$$

given by

$$
\left(\sigma, \xi_{1}\right) \mapsto \sigma \cdot \xi_{1}=\left(\sigma_{1} \wedge \xi_{1},\left((\partial-\bar{\partial}) \sigma_{01}\right) \wedge \xi_{1}-\frac{1}{2}(\partial-\bar{\partial})\left(\sigma_{01} \wedge \xi_{1}\right)\right)
$$

Proposition 4.9 The above bilinear map induces a bilinear map

$$
H_{\mathrm{BC}}^{p, q}\left(\mathcal{U}, U_{0}\right) \times H_{\mathrm{A}}^{n-p, n-q}\left(U_{1}\right) \stackrel{\cdot}{\longrightarrow} H_{D}^{2 n}\left(\mathcal{U}, U_{0}\right) .
$$

Proof: We need to show that

(1) If $\sigma \in Z_{\mathrm{BC}}^{p, q}\left(\mathcal{U}, U_{0}\right)$ and $\xi_{1} \in Z_{\mathrm{A}}^{n-p, n-q}\left(U_{1}\right)$, then $\sigma \cdot \xi_{1} \in Z^{2 n}\left(\mathcal{U}, U_{0}\right)$.

(2) If $\sigma \in B_{\mathrm{BC}}^{p, q}\left(\mathcal{U}, U_{0}\right)$ and $\xi_{1} \in Z_{\mathrm{A}}^{n-p, n-q}\left(U_{1}\right)$, then $\sigma \cdot \xi_{1} \in B^{2 n}\left(\mathcal{U}, U_{0}\right)$.

(3) If $\sigma \in Z_{\mathrm{BC}}^{p, q}\left(\mathcal{U}, U_{0}\right)$ and $\xi_{1} \in B_{\mathrm{A}}^{n-p, n-q}\left(U_{1}\right)$, then $\sigma \cdot \xi_{1} \in B^{2 n}\left(\mathcal{U}, U_{0}\right)$.

In the above $Z_{\mathrm{A}}^{n-p, n-q}\left(U_{1}\right)$ and $B_{\mathrm{A}}^{n-p, n-q}\left(U_{1}\right)$ denote the Aeppli cocycles and coboundaries on $U_{1}$ and $Z^{2 n}\left(\mathcal{U}, U_{0}\right)$ and $B^{2 n}\left(\mathcal{U}, U_{0}\right)$ the relative de Rham cocycles and coboundaries.

To prove (1), take $\sigma \in Z_{\mathrm{BC}}^{p, q}\left(\mathcal{U}, U_{0}\right)$ and $\xi_{1} \in Z_{\mathrm{A}}^{n-p, n-q}\left(U_{1}\right)$. Thus $d \sigma_{1}=0, \sigma_{1}-\bar{\partial} \partial \sigma_{01}=$ 0 and $\bar{\partial} \partial \xi_{1}=0$. We have $d\left(\sigma \cdot \xi_{1}\right)_{1}=d\left(\sigma_{1} \wedge \xi_{1}\right)=0$ by the degree reason. By (4.4) and the degree reason we compute $\left(\sigma \cdot \xi_{1}\right)_{1}-d\left(\sigma \cdot \xi_{1}\right)_{01}=0$. Thus $\sigma \cdot \xi_{1} \in Z^{2 n}\left(\mathcal{U}, U_{0}\right)$.

To prove (2), take $\sigma \in B_{\mathrm{BC}}^{p, q}\left(\mathcal{U}, U_{0}\right)$ and $\xi \in Z_{\mathrm{A}}^{n-p, n-q}\left(U_{1}\right)$. Thus $\sigma_{1}=\bar{\partial} \partial \tau_{1}$ and $\sigma_{01}=\tau_{1}-\bar{\partial} \tau_{01}^{(1)}-\partial \tau_{01}^{(2)}$ (cf. Remark 4.5). Also $\bar{\partial} \partial \xi_{1}=0$. Then we may write, again by the degree reason,

$$
\left(\sigma \cdot \xi_{1}\right)_{1}=d\left(\alpha_{1}^{(1)}+\alpha_{1}^{(2)}\right),
$$

where $\alpha_{1}^{(k)}, k=1,2$, are as in the proof of Proposition 4.3. In (4.6), we have $\alpha_{01}^{(k l)}=0$ as $\tau_{0}=0$. Also $\bar{\partial} \gamma_{01}^{(11)}=d \gamma_{01}^{(11)}$ and $\partial \gamma_{01}^{(22)}=d \gamma_{01}^{(22)}$ as $\gamma_{01}^{(11)}$ and $\gamma_{01}^{(22)}$ are $(n, n-2)$ and $(n-2, n)$-forms, respectively. Thus we have

$$
\left(\sigma \cdot \xi_{1}\right)_{01}=\alpha_{1}^{(1)}+\alpha_{1}^{(2)}-d\left(\gamma_{01}^{(11)}+\gamma_{01}+\gamma_{01}^{(22)}\right),
$$


where $\gamma_{01}=\gamma_{01}^{(12)}=\gamma_{01}^{(21)}$. This shows $\sigma \cdot \xi_{1} \in B^{2 n}\left(\mathcal{U}, U_{0}\right)$.

To prove (3), take $\sigma \in Z_{\mathrm{BC}}^{p, q}\left(\mathcal{U}, U_{0}\right)$ and $\xi_{1} \in B_{\mathrm{A}}^{n-p, n-q}\left(U_{1}\right)$. Thus $d \sigma_{1}=0, \sigma_{1}-$ $\bar{\partial} \partial \sigma_{01}=0$ and $\xi_{1}=\bar{\partial} \eta_{1}^{(1)}+\partial \eta_{1}^{(2)}$. Then we have, with $\beta_{1}^{(k)}$ and $\beta_{01}^{(k l)}$ as in the proof of Proposition 4.3,

$$
\left(\sigma \cdot \xi_{1}\right)_{1}=d\left(\beta_{1}^{(1)}+\beta_{1}^{(2)}\right), \quad\left(\sigma \cdot \xi_{1}\right)_{01}=\beta_{1}^{(1)}+\beta_{1}^{(2)}-d\left(\beta_{01}^{(11)}+\beta_{01}+\beta_{01}^{(22)}\right),
$$

where $\beta_{01}=\beta_{01}^{(12)}=\beta_{01}^{(21)}$, which shows $\sigma \cdot \xi_{1} \in B^{2 n}\left(\mathcal{U}, U_{0}\right)$.

\subsection{Integration}

We may define integration on Čech-Bott-Chern or Čech-Aeppli cohomology by making use of the integration theory on Čech-de Rham cohomology. The Čech-de Rham cohomology and its integration theory may be developed for an arbitrary covering of a $C^{\infty}$ manifold. Here we briefly recall the theory in our situation and refer to [16] and [21] for the general case and details.

Let $M$ be a complex manifold of dimension $n$ and $\mathcal{U}=\left\{U_{0}, U_{1}\right\}$ a covering of $M$ as before. The Čech-de Rham cohomology $H_{D}^{r}(\mathcal{U})$ is defined as in Example 3.10.1. Let $\left\{R_{0}, R_{1}\right\}$ be a "honeycomb system" adapted to $\mathcal{U}$. In our case we may assume that each $R_{i}$ is a closed real $2 n$-dimensional manifold with $C^{\infty}$ boundary $\partial R_{i}$ in $U_{i}, i=0,1$, such that $R_{0} \cup R_{1}=M$ and Int $R_{0} \cap \operatorname{Int} R_{1}=\emptyset$, where "Int" means the interior. We set $R_{01}=R_{0} \cap R_{1}$, which coincides with $\partial R_{0}$ and is endowed with the orientation of $\partial R_{0}$ so that $R_{01}=\partial R_{0}=-\partial R_{1}$, as oriented manifolds.

Suppose $M$ is compact. Then we may assume that $R_{0}$ and $R_{1}$ are compact and we have the integration

$$
\int_{M}: \mathcal{E}^{2 n}(\mathcal{U}) \longrightarrow \mathbb{C} \quad \text { given by } \int_{M} \zeta=\int_{R_{0}} \zeta_{0}+\int_{R_{1}} \zeta_{1}+\int_{R_{01}} \zeta_{01},
$$

which induces the integration on $H_{D}^{2 n}(\mathcal{U})$. It is compatible with the integration on the de Rham cohomology $H_{d}^{2 n}(M)$ via the isomorphism (3.11).

In the relative case we proceed as follows. Thus let $S$ be a closed set in $M$. Let $U_{0}=M \backslash S$ and $U_{1}$ a neighborhood of $S$ and consider the covering $\mathcal{U}=\left\{U_{0}, U_{1}\right\}$ of $M$ as before. We have the relative de Rham cohomology $H_{D}^{r}\left(\mathcal{U}, U_{0}\right)$ as in Example 3.10.1. Suppose $S$ is compact ( $M$ may not be). Let $R_{1}$ be a $2 n$-dimensional compact submanifold of $M$ with $C^{\infty}$ boundary containing $S$ in its interior and set $R_{01}=-\partial R_{1}$. Then we have the integration

$$
\int_{M}: H_{D}^{2 n}\left(\mathcal{U}, U_{0}\right) \longrightarrow \mathbb{C} \quad \text { induced by } \int_{M} \zeta=\int_{R_{1}} \zeta_{1}+\int_{R_{01}} \zeta_{01} .
$$

Now we consider the Aeppli case. First suppose $M$ is compact. Then the integration on $H_{\mathrm{A}}^{n, n}(M)$ is defined using the identity $H_{\mathrm{A}}^{n, n}(M)=H_{d}^{2 n}(M)$. The integration on the ČechAeppli cohomology is defined using Proposition 3.23. Namely, we have the integration

$$
\int_{M}: H_{\mathrm{A}}^{n, n}(\mathcal{U}) \longrightarrow \mathbb{C} \quad \text { induced by } \int_{M} \xi=\int_{R_{0}} \xi_{0}+\int_{R_{1}} \xi_{1}+\int_{R_{01}}\left(\xi_{01}^{(1)}+\xi_{01}^{(2)}\right) .
$$


In the relative case we may use Proposition 3.25. Thus if $S$ is compact, we have the integration

$$
\int_{M}: H_{\mathrm{A}}^{n, n}\left(\mathcal{U}, U_{0}\right) \longrightarrow \mathbb{C} \quad \text { induced by } \int_{M} \xi=\int_{R_{1}} \xi_{1}+\int_{R_{01}}\left(\xi_{01}^{(1)}+\xi_{01}^{(2)}\right) .
$$

Remark 4.12 Noting that there is a canonical morphism $H_{\bar{\vartheta}}^{n, q}(\mathcal{U}) \rightarrow H_{D}^{n+q}(\mathcal{U})$, we may define the integration on $H_{\mathrm{BC}}^{n, n}(\mathcal{U})$ as the composition

$$
H_{\mathrm{BC}}^{n, n}(\mathcal{U}) \longrightarrow H_{\bar{\vartheta}}^{n, n}(\mathcal{U}) \longrightarrow H_{D}^{2 n}(\mathcal{U}) \stackrel{\int_{M}}{\longrightarrow} \mathbb{C}
$$

\subsection{Duality morphisms}

If $M$ is compact, we have a bilinear map

$$
H_{\mathrm{BC}}^{p, q}(M) \times H_{\mathrm{A}}^{n-p, n-q}(M) \stackrel{\wedge}{\longrightarrow} H_{\mathrm{A}}^{n, n}(M) \stackrel{\int_{M}}{\longrightarrow} \mathbb{C},
$$

which is non-degenerate so that we have an isomorphism (cf. [20])

$$
\Phi: H_{\mathrm{BC}}^{p, q}(M) \stackrel{\sim}{\longrightarrow} H_{\mathrm{A}}^{n-p, n-q}(M)^{*} .
$$

Now we consider the duality between Čech-Bott-Chern and Čech-Aeppli cohomologies. If $M$ is compact, we have a pairing (cf. Proposition 4.3 and (4.11)) :

$$
H_{\mathrm{BC}}^{p, q}(\mathcal{U}) \times H_{\mathrm{A}}^{n-p, n-q}(\mathcal{U}) \stackrel{\smile}{\longrightarrow} H_{\mathrm{A}}^{n, n}(\mathcal{U}) \stackrel{\int_{M}}{\longrightarrow} \mathbb{C},
$$

which is compatible with (4.13). Thus we have an isomorphism

$$
\Phi: H_{\mathrm{BC}}^{p, q}(\mathcal{U}) \stackrel{\sim}{\longrightarrow} H_{\mathrm{A}}^{n-p, n-q}(\mathcal{U})^{*} \simeq H_{\mathrm{A}}^{n-p, n-q}(M)^{*} .
$$

In the relative case, suppose $S$ is compact. We then have a pairing (cf. Proposition 4.9 and (4.10)) :

$$
H_{\mathrm{BC}}^{p, q}\left(\mathcal{U}, U_{0}\right) \times H_{\mathrm{A}}^{n-p, n-q}\left(U_{1}\right) \stackrel{\cdot}{\longrightarrow} H_{D}^{2 n}\left(\mathcal{U}, U_{0}\right) \stackrel{\int_{M}}{\longrightarrow} \mathbb{C},
$$

which induces a morphism $H_{\mathrm{BC}}^{p, q}\left(\mathcal{U}, U_{0}\right) \rightarrow H_{\mathrm{A}}^{n-p, n-q}\left(U_{1}\right)^{*}$. If we set

$$
H_{\mathrm{A}}^{n-p, n-q}[S]=\underset{U_{1} \supset S}{\lim _{\mathrm{A}}} H_{\mathrm{A}}^{n-p, n-q}\left(U_{1}\right)
$$

the inductive limit over the open neighborhoods $U_{1}$ of $S$, we have a morphism (cf. Corollary 3.8)

$$
\Psi: H_{\mathrm{BC}}^{p, q}\left(\mathcal{U}, U_{0}\right) \longrightarrow H_{\mathrm{A}}^{n-p, n-q}[S]^{*}
$$

Proposition 4.16 If $M$ is compact, we have the following commutative diagram:

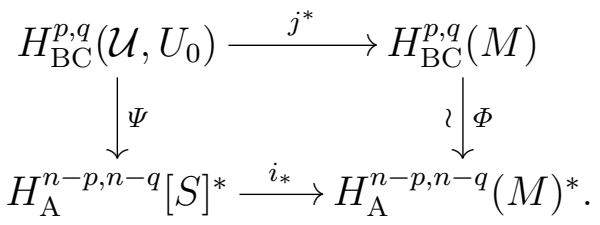




\section{Characteristic classes in Čech-Bott-Chern cohomol- ogy}

\subsection{Chern forms}

We start by briefly recalling the Chern-Weil theory of characteristic classes. For details we refer to [5], [8], [19] and [21].

Let $M$ be a $C^{\infty}$ manifold and $E \rightarrow M$ a $C^{\infty}$ complex vector bundle of rank $l$. For an open set $U$ in $M$, we denote by $\mathcal{E}^{r}(U, E)$ the vector space of $E$-valued $r$-forms on $U$, i.e., $C^{\infty}$ sections of the bundle $\bigwedge^{r} \tau^{*} \otimes E$ on $U$, where $\tau=T_{\mathbb{R}}^{c} M$ denotes the complexification of the real tangent bundle $T_{\mathbb{R}} M$ of $M$.

A connection for $E$ is a $\mathbb{C}$-linear map

$$
\nabla: \mathcal{E}^{0}(M, E) \longrightarrow \mathcal{E}^{1}(M, E)
$$

which is a derivation: $\nabla(f s)=d f \otimes s+f \nabla(s)$ for $f \in \mathcal{E}^{0}(M), s \in \mathcal{E}^{0}(M, E)$. For a vector field $u$, we denote $\nabla(s)(u)$ by $\nabla_{u}(s)$, which is in $\mathcal{E}^{0}(M, E)$. A connection $\nabla$ induces a derivation $\nabla: \mathcal{E}^{1}(M, E) \rightarrow \mathcal{E}^{2}(M, E)$ and the composition

$$
K=\nabla \circ \nabla: \mathcal{E}^{0}(M, E) \longrightarrow \mathcal{E}^{2}(M, E)
$$

is the curvature of $\nabla$. It is $\mathcal{E}^{0}(M)$-linear so that it can be thought of as a 2 -form with coefficients in $\operatorname{Hom}(E, E)$. Thus, for the $p$-th elementary invariant polynomial $\sigma_{p}, p=$ $1, \ldots, l, \sigma_{p}(K)$ is a $2 p$-form on $M$, which is shown to be closed. The $p$-th Chern form of $\nabla$ is defined by

$$
c^{p}(\nabla)=\left(\frac{\sqrt{-1}}{2 \pi}\right)^{p} \sigma_{p}(K) .
$$

More generally, if $\varphi$ is an invariant polynomial homogeneous of degree $k$, we have a well-defined $2 k$-form $\varphi(K)$, which is closed. By a slight abuse of notation, we set

$$
\varphi(\nabla)=\left(\frac{\sqrt{-1}}{2 \pi}\right)^{k} \varphi(K)
$$

Alternatively, for such $\varphi$, there is a polynomial $P$ such that $\varphi=P\left(\sigma_{1}, \sigma_{2}, \ldots\right)$. Then $\varphi(\nabla)=P\left(c^{1}(\nabla), c^{2}(\nabla), \ldots\right)$.

Noting that a connection is a local operator, we have local representations of the connection and curvature. For a (local) frame $\boldsymbol{e}=\left(e_{1}, \ldots, e_{l}\right)$ of $E$, the connection matrix $\theta=\left(\theta_{i j}\right)$ is determined by $\nabla e_{i}=\sum_{i=1}^{l} \theta_{j i} e_{j}$. Also the curvature matrix $\kappa=\left(\kappa_{i j}\right)$ is determined by $K e_{i}=\sum_{i=1}^{l} \kappa_{j i} e_{j}$. We then have

$$
\kappa=d \theta+\theta \wedge \theta
$$

By a frame change $\boldsymbol{e}^{\prime}=\boldsymbol{e} A$, the connection and curvature matrices become

$$
\theta^{\prime}=A^{-1} \cdot d A+A^{-1} \theta A, \quad \kappa^{\prime}=A^{-1} \kappa A .
$$

The form $\varphi(\nabla)$ above is locally given by $\left(\frac{\sqrt{-1}}{2 \pi}\right)^{k} \varphi(\kappa)$. 
Bott difference form : Let $\varphi$ be as above. For two connections $\nabla$ and $\nabla^{\prime}$ for $E$, there exists a $(2 k-1)$-form $\varphi\left(\nabla, \nabla^{\prime}\right)$ such that $\varphi\left(\nabla^{\prime}, \nabla\right)=-\varphi\left(\nabla, \nabla^{\prime}\right)$ and that

$$
d \varphi\left(\nabla, \nabla^{\prime}\right)=\varphi\left(\nabla^{\prime}\right)-\varphi(\nabla) .
$$

As to the construction of such forms, we refer to [7], [8] and [21]. One of the consequences of this is that the class of $[\varphi(\nabla)]$ in $H_{d}^{2 k}(M)$ is independent of the choice of the connection $\nabla$ for $E$. The class will be denoted by $\varphi(E)$. In particular the class of $c^{p}(\nabla)$ is the $p$-th Chern class $c^{p}(E)$ of $E$.

\subsection{Metric connections}

Connections of type $(\mathbf{1 , 0 )}$ : Now let $M$ be a complex manifold of dimension $n$ and $E \rightarrow M$ a holomorphic vector bundle of rank $l$. A connection $\nabla$ for $E$ is of type $(1,0)$ (a $(1,0)$-connection for short) if the entries of the connection matrix with respect to a holomorphic frame are forms of type $(1,0)$. Note that this property does not depend on the choice of the holomorphic frame and that every holomorphic vector bundle admits a $(1,0)$-connection. If $\nabla$ is a $(1,0)$-connection for $E$, we may write its curvature $K$ as

$$
K=K^{2,0}+K^{1,1}
$$

with $K^{2,0}$ and $K^{1,1}$ a $(2,0)$-form and a $(1,1)$-form with coefficients in $\operatorname{Hom}(E, E)$. Locally, if $\theta$ and $\kappa$ are the connection and curvature matrices of $\nabla$ with respect to a holomorphic frame, we can decompose as $\kappa=\kappa^{2,0}+\kappa^{1,1}$ according to the types, and $K^{2,0}$ and $K^{1,1}$ are represented by (cf. (5.1))

$$
\kappa^{2,0}=\partial \theta+\theta \wedge \theta \quad \text { and } \quad \kappa^{1,1}=\bar{\partial} \theta .
$$

Thus $K^{1,1}$, being locally $\bar{\partial}$-exact, is a $\bar{\partial}$-closed $(1,1)$-form with coefficients in $\operatorname{Hom}(E, E)$.

Atiyah forms: In the above situation, for a $(1,0)$-connection $\nabla$, we have the $\bar{\partial}$-closed $(p, p)$-form $\sigma_{p}\left(K^{1,1}\right)$ on $M$, which is locally given by $\sigma_{p}\left(\kappa^{1,1}\right)$. The $p$-th Atiyah form of $\nabla$ is defined by (cf. [2])

$$
a^{p}(\nabla)=\left(\frac{\sqrt{-1}}{2 \pi}\right)^{p} \sigma_{p}\left(K^{1,1}\right) .
$$

If $\nabla$ is a $(1,0)$-connection, the $p$-th Chern form $c^{p}(\nabla)$ is a $2 p$-form having components of types $(2 p, 0), \ldots,(p, p)$. The Atiyah form $a^{p}(\nabla)$ is the $(p, p)$-component of $c^{p}(\nabla)$. In particular, $a^{n}(\nabla)=c^{n}(\nabla)$.

If $\varphi$ is an invariant polynomial homogeneous of degree $k$ and if $\nabla$ is a $(1,0)$-connection, $\varphi(\nabla)$ is a closed $2 k$-form having components of types $(2 k, 0), \ldots,(k, k)$. We denote the $(k, k)$-component of $\varphi(\nabla)$ by $\varphi^{\mathrm{A}}(\nabla)$ and call it the Atiyah form of $\nabla$ with respect to $\varphi$. If $\varphi=P\left(\sigma_{1}, \sigma_{2}, \ldots\right)$, then $\varphi^{\mathrm{A}}(\nabla)=P\left(a^{1}(\nabla), a^{2}(\nabla), \ldots\right)$, which is a $\bar{\partial}$-closed $(k, k)$-form on $M$.

If we have two $(1,0)$-connections $\nabla$ and $\nabla^{\prime}$ for $E$, we have the difference form $\varphi^{\mathrm{A}}\left(\nabla, \nabla^{\prime}\right)$. It is the $(k, k-1)$-component of $\varphi\left(\nabla, \nabla^{\prime}\right)$ and satisfies

$$
\bar{\partial} \varphi^{\mathrm{A}}\left(\nabla, \nabla^{\prime}\right)=\varphi^{\mathrm{A}}\left(\nabla^{\prime}\right)-\varphi^{\mathrm{A}}(\nabla) .
$$

Thus, if $\nabla$ is a $(1,0)$-connection for $E$, the class of $\varphi^{\mathrm{A}}(\nabla)$ in $H_{\bar{\partial}}^{k, k}(M)$ does not depend on the choice of $\nabla$. It is denoted by $\varphi^{\mathrm{A}}(E)$ and is called the Atiyah class of $E$ with respect to $\varphi$. In particular, the class of $a^{p}(\nabla)$ in $H_{\bar{\partial}}^{p, p}(M)$ is the $p$-th Atiyah class $a^{p}(E)$ of $E$. 
Hermitian connections: A Hermitian vector bundle $(E, h)$ is a $C^{\infty}$ complex vector bundle $E$ together with a Hermitian metric $h$ on $E$. A connection $\nabla$ for $E$ is an $h$ connection if it is compatible with $h$ :

$$
d h(s, t)=h(\nabla s, t)+h(s, \nabla t) \quad \text { for } \quad s, t \in \mathcal{E}^{0}(M, E) .
$$

A connection $\nabla$ for $E$ is Hermitian, if it is an $h$-connection for some $h$. Note that every Hermitian vector bundle $(E, h)$ admits an $h$-connection. Thus every complex vector bundle admits a Hermitian connection.

Metric connections : Let $M$ be a complex manifold and $E \rightarrow M$ a holomorphic vector bundle. Recall that, for any Hermitian metric $h$ on $E$, there exists a unique $h$-connection of type $(1,0)$. We call such a connection the $h$-metric connection. Let $\nabla$ be the $h$-metric connection. Let $\boldsymbol{e}=\left(e_{1}, \ldots, e_{l}\right)$ be a (local) holomorphic frame and $\theta$ the connection matrix of $\nabla$ with respect to $\boldsymbol{e}$. We also set $H=\left(h_{i j}\right), h_{i j}=h\left(e_{i}, e_{j}\right)$. Then the connection matrix with respect to $\boldsymbol{e}$ is given by

$$
\theta={ }^{t} H^{-1} \cdot \partial^{t} H
$$

We call a Hermitian connection of type $(1,0)$ a metric connection. From (5.3) we see that, if $\nabla$ is a metric connection for $E$, its curvature matrix $\kappa$ with respect to a holomorphic frame is of type $(1,1)$ :

$$
\kappa=\kappa^{1,1}=\bar{\partial} \theta .
$$

Thus we have:

Proposition 5.5 Let $E$ be a holomorphic vector bundle. If $\nabla$ is a metric connection for $E$, the Chern forms and the Atiyah forms are the same:

$$
\varphi(\nabla)=\varphi^{\mathrm{A}}(\nabla)
$$

Thus they are simultaneously $d$ and $\bar{\partial}$-closed.

Remark 5.6 1. For two metric connections $\nabla$ and $\nabla^{\prime}$, we also have

$$
\varphi\left(\nabla, \nabla^{\prime}\right)=\varphi^{\mathrm{A}}\left(\nabla, \nabla^{\prime}\right)
$$

2. Let $(E, h)$ be a Hermitian line bundle and $s$ a non-vanishing holomorphic section of $E$. We may think of $s$ as a frame and set $N(s)=H=h(s, s)$, the square of the norm of $s$. Then, by (5.3) and (5.4), the first Chern form of the $h$-metric connection $\nabla$ is given by

$$
c^{1}(\nabla)=\frac{\sqrt{-1}}{2 \pi} \kappa=\frac{\sqrt{-1}}{2 \pi} \bar{\partial} \partial \log N(s),
$$

which is also equal to $a^{1}(\nabla)$. 


\subsection{Bott-Chern classes}

Let $E \rightarrow M$ be a holomorphic vector bundle on a complex manifold. For an invariant polynomial $\varphi$ and a Hermitian metric $h$ on $E$ we set $\varphi(E, h)=\varphi(\nabla)$, where $\nabla$ is the $h$-metric connection. If we have two metrics $h$ and $h^{\prime}$ on $E$, we have the Bott-Chern form $\varphi\left(E, h, h^{\prime}\right)$ which relates $\varphi(E, h)$ and $\varphi\left(E, h^{\prime}\right)$. It was first introduced in [8] and then has been studied by a number of authors via different approaches (cf. [6], [11], [12], [14] and references therein). We may summarize what we need in our situation as:

Theorem 5.7 Let $\varphi$ be an invariant polynomial homogeneous of degree $k$. There is a unique way of assigning to $\left(E, h, h^{\prime}\right)$ a $(k-1, k-1)$-form $\varphi\left(E, h, h^{\prime}\right)$ so that

(1) $\bar{\partial} \partial \varphi\left(E, h, h^{\prime}\right)=\varphi\left(E, h^{\prime}\right)-\varphi(E, h)$,

(2) $f^{*} \varphi\left(E, h, h^{\prime}\right)=\varphi\left(f^{*}\left(E, h, h^{\prime}\right)\right)$ for every holomorphic map $f: M^{\prime} \rightarrow M$,

(3) $\varphi\left(E, h, h^{\prime}\right)=0$, if $h=h^{\prime}$.

In particular from (1) above we see that the class of $\varphi(E, h)$ in $H_{\mathrm{BC}}^{k, k}(M)$ does not depend on the choice of the metric $h$.

Definition 5.8 The Bott-Chern class $\varphi_{\mathrm{BC}}(E)$ of $E$ with respect to $\varphi$ is the class in $H_{\mathrm{BC}}^{k, k}(M)$ of $\varphi(E, h)$ for some Hermitian metric $h$ on $E$.

In the sequel we denote $\varphi(E, h)$ and $\varphi\left(E, h, h^{\prime}\right)$ simply by $\varphi(h)$ and $\varphi\left(h, h^{\prime}\right)$, if the bundle under consideration is understood.

Remark 5.9 1. The Bott-Chern Chern class and the Bott-Chern Atiyah class are the same: $c_{\mathrm{BC}}^{p}(E)=a_{\mathrm{BC}}^{p}(E)$ in $H_{\mathrm{BC}}^{p, p}(M)$. It goes to the Atiyah class $a^{p}(E)$ by the first morphism in (2.2) and to the Chern class $c^{p}(E)$ by the second.

2. Suppose $E$ is a line bundle with Hermitian metrics $h$ and $h^{\prime}$. For a non-vanishing section $s$, we set $N(s)=h(s, s)$ and $N^{\prime}(s)=h^{\prime}(s, s)$. Then, for $\varphi=c^{1}$, we have

$$
c^{1}\left(h, h^{\prime}\right)=\frac{\sqrt{-1}}{2 \pi} \log \frac{N^{\prime}(s)}{N(s)} .
$$

3. In general, if $h_{0}$ and $h_{1}$ are Hermitian metrics on $E$ defined on open sets $U_{0}$ and $U_{1}$ in $M$, respectively, the form $\varphi\left(h_{0}, h_{1}\right)$ as above is defined on $U_{01}=U_{0} \cap U_{1}$.

\subsection{Characteristic classes in Čech-Bott-Chern cohomology}

Let $E$ be a holomorphic vector bundle on a complex manifold $M$ and $\mathcal{U}=\left\{U_{0}, U_{1}\right\}$ an open covering of $M$. For $i=0,1$, let $h_{i}$ be a metric for $E$ on $U_{i}$. If $\varphi$ is an invariant polynomial homogeneous of degree $k$, the cochain

$$
\varphi\left(h_{*}\right)=\left(\varphi\left(h_{0}\right), \varphi\left(h_{1}\right), \varphi\left(h_{0}, h_{1}\right)\right)
$$

in

$$
\mathcal{E}_{\mathrm{BC}}^{k, k}(\mathcal{U})=\mathcal{E}^{k, k}\left(U_{0}\right) \oplus \mathcal{E}^{k, k}\left(U_{1}\right) \oplus \mathcal{E}^{k-1, k-1}\left(U_{01}\right) .
$$

is a cocycle. Thus we have a class $\left[\varphi\left(h_{*}\right)\right]$ in $H_{\mathrm{BC}}^{k, k}(\mathcal{U})$. 
Proposition 5.10 The class does not depend on the choice of the metrics. Moreover it corresponds to $\varphi_{B C}(E) \in H_{\mathrm{BC}}^{k, k}(M)$ via the isomorphism (3.17).

Proof: We recall (cf. [11, Proposition 4.23]) that, for three metrics $\left(h, h^{\prime}, h^{\prime \prime}\right)$, there exist a $(k-1, k-2)$-form $\varphi^{(1)}\left(h, h^{\prime}, h^{\prime \prime}\right)$ and a $(k-2, k-1)$-form $\varphi^{(2)}\left(h, h^{\prime}, h^{\prime \prime}\right)$ such that

$$
\varphi\left(h^{\prime}, h^{\prime \prime}\right)-\varphi\left(h, h^{\prime \prime}\right)+\varphi\left(h, h^{\prime}\right)+\bar{\partial} \varphi^{(1)}\left(h, h^{\prime}, h^{\prime \prime}\right)+\partial \varphi^{(2)}\left(h, h^{\prime}, h^{\prime \prime}\right)=0 .
$$

Let $h_{0}^{\prime}$ be another metric on $E$ on $U_{0}$ and set

$$
\varphi\left(h_{*}^{\prime}\right)=\left(\varphi\left(h_{0}^{\prime}\right), \varphi\left(h_{1}\right), \varphi\left(h_{0}^{\prime}, h_{1}\right)\right) .
$$

Then we have

$$
\begin{aligned}
\varphi\left(h_{*}^{\prime}\right)-\varphi\left(h_{*}\right) & =\left(\varphi\left(h_{0}^{\prime}\right)-\varphi\left(h_{0}\right), 0, \varphi\left(h_{0}^{\prime}, h_{1}\right)-\varphi\left(h_{0}, h_{1}\right)\right) \\
& =\left(\bar{\partial} \partial \varphi\left(h_{0}, h_{0}^{\prime}\right), 0,-\varphi\left(h_{0}, h_{0}^{\prime}\right)-\bar{\partial} \varphi^{(1)}\left(h_{0}, h_{0}^{\prime}, h_{1}\right)-\partial \varphi^{(2)}\left(h_{0}, h_{0}^{\prime}, h_{1}\right),\right.
\end{aligned}
$$

which is in $B_{\mathrm{BC}}^{k, k}(\mathcal{U})($ cf. $(3.15))$ so that $\left[\varphi\left(h_{*}^{\prime}\right)\right]=\left[\varphi\left(h_{*}\right)\right]$.

Likewise Let $h_{1}^{\prime}$ be another metric on $E$ on $U_{1}$ and set

$$
\varphi\left(h_{*}^{\prime}\right)=\left(\varphi\left(h_{0}\right), \varphi\left(h_{1}^{\prime}\right), \varphi\left(h_{0}, h_{1}^{\prime}\right)\right) .
$$

Then we have

$$
\begin{aligned}
\varphi\left(h_{*}^{\prime}\right)-\varphi\left(h_{*}\right) & =\left(0, \varphi\left(h_{1}^{\prime}\right)-\varphi\left(h_{1}\right), \varphi\left(h_{0}, h_{1}^{\prime}\right)-\varphi\left(h_{0}, h_{1}\right)\right) \\
& =\left(0, \bar{\partial} \partial \varphi\left(h_{1}, h_{1}^{\prime}\right), \varphi\left(h_{1}, h_{1}^{\prime}\right)+\bar{\partial} \varphi^{(1)}\left(h_{0}, h_{1}, h_{1}^{\prime}\right)+\partial \varphi^{(2)}\left(h_{0}, h_{1}, h_{1}^{\prime}\right),\right.
\end{aligned}
$$

which is in $B_{\mathrm{BC}}^{k, k}(\mathcal{U})$ (in fact in $B_{\mathrm{BC}}^{k, k}\left(\mathcal{U}, U_{0}\right)$ ).

The last part can be seen by comparing with the class defined by a global metric.

\section{A vanishing theorem}

\subsection{Actions of distributions}

Let $M$ be a complex manifold of dimension $n$ and $F$ a non-singular distribution of dimension $p$, i.e., a subbundle of $T M$ of rank $p$.

Definition 6.1 A (holomorphic) action of $F$ on a holomorphic vector bundle $E$ over $M$ is a $\mathbb{C}$-bilinear map

$$
\alpha: \mathcal{E}^{0}(M, F) \times \mathcal{E}^{0}(M, E) \longrightarrow \mathcal{E}^{0}(M, E)
$$

satisfying the following conditions, for $f \in \mathcal{E}^{0}(M), u \in \mathcal{E}^{0}(M, F), s \in \mathcal{E}^{0}(M, E)$ :

(1) $\alpha(f u, s)=f \alpha(u, s)$,

(2) $\alpha(u, f s)=u(f) s+f \alpha(u, s)$ and

(3) $\alpha(u, s)$ is holomorphic whenever $u$ and $s$ are.

A vector bundle $E$ with an action of $F$ is called an $F$-bundle. 
Definition 6.2 Let $E$ be an $F$-bundle with action $\alpha$. An $F$-connection for $E$ is a $(1,0)$ connection $\nabla$ with

$$
\nabla_{u}(s)=\alpha(u, s), \quad \text { for } s \in \mathcal{E}^{0}(M, E), u \in \mathcal{E}^{0}(M, F) .
$$

From the fact that an action is a local operation, we see that an $F$-bundle always admits an $F$-connection.

We note that the above material can be equivalently treated in terms of partial holomorphic connections instead of actions (cf. [2]), the condition (4) in Remark 6.5.1 below corresponding to the fact that the partial connection is flat. We have the following Bott type vanishing theorem for $F$-connections, which is proved in [2, Theorem 6.10] in the context of partial connections.

Theorem 6.3 Let $M$ and $F$ be as above. Let $E$ be an $F$-bundle and $\nabla$ an $F$-connection for $E$. For an invariant polynomial $\varphi$ homogeneous of degree $k>n-p$, we have:

$$
\varphi^{\mathrm{A}}(\nabla)=0 .
$$

Corollary 6.4 If, in addition, $\nabla$ is Hermitian,

$$
\varphi(\nabla)=0 .
$$

Remark 6.5 1. For two connections $\nabla$ and $\nabla^{\prime}$ and an invariant polynomial $\varphi$ as in Theorem 6.3 , we have the vanishing $\varphi^{\mathrm{A}}\left(\nabla, \nabla^{\prime}\right)=0$. Thus if, in addition, $\nabla$ and $\nabla^{\prime}$ are Hermitian, $\varphi\left(\nabla, \nabla^{\prime}\right)=0$ (cf. Remark 5.6.1). However if $\nabla$ and $\nabla^{\prime}$ are Hermitian with respect to different metrics $h$ and $h^{\prime}$, the form $\varphi\left(h, h^{\prime}\right)$ does not vanish in general, as the following example, which was communicated to us by the referee, shows.

Let $E=M \times \mathbb{C}$ be the product bundle and $F=T M$ so that $p=n$. Then there is a natural action of $F$ on $E$ (the action of vector fields on functions). The exterior derivative $d$ is an $F$-connection for $E$. It is also the metric connection with respect to any constant metric on $E$, i.e., a metric $h$ given by $h(e, e)=a$ (a positive real number) with $e$ the frame of $E$ defined by $x \mapsto(x, 1)$. Note that for $\varphi=c^{1}, \operatorname{deg} \varphi=1>n-p=0$. However, for the two metrics $h$ and $h^{\prime}$ given by $h(e, e)=1$ and $h^{\prime}(e, e)=a \neq 1$, we have (cf. Remark 5.9.3)

$$
c^{1}\left(h, h^{\prime}\right)=\frac{\sqrt{-1}}{2 \pi} \log a \neq 0 .
$$

2. In Theorem 6.3 , if $F$ is involutive and if the action satisfies

(4) $\alpha([u, v], s)=\alpha(u, \alpha(v, s))-\alpha(v, \alpha(u, s))$,

we have $\varphi(\nabla)=0$ for $\varphi$ with $k>n-p$. This is usually referred to as the Bott vanishing theorem.

3. Let $h$ be a Hermitian metric on $E$. Under the condition of Corollary 6.4, we have

$$
u(h(s, t))=h\left(\nabla_{u}(s), t\right),
$$

for $u$ in $A^{0}(M, F)$ and a holomorphic section $t$ of $E$.

4. Some special cases of this Bott type vanishing theorem are proved in [13]. 


\subsection{Action on the normal bundle of an invariant submanifold}

Let $M$ be a complex manifold of dimension $n$ and $V$ a complex submanifold of dimension $d$ of $M$. Let $N_{V}$ be the normal bundle of $V$ in $M$ so that we have the exact sequence

$$
\left.0 \longrightarrow T V \longrightarrow T M\right|_{V} \stackrel{\pi}{\longrightarrow} N_{V} \longrightarrow 0
$$

Let $F$ be a distribution of dimension $p$ on $M$. We say that $F$ leaves $V$ invariant if $\left.F\right|_{V} \subset T V$. In this case we set $F_{V}=\left.F\right|_{V}$, which is a distribution of dimension $p$ on $V$. The following is proved in [17] (see also [18]) for the case of foliations. In fact the involutivity of $F$ is not necessary and a proof is given in [2] in terms of partial connections and in [24] in terms of actions.

Theorem 6.7 In the above situation, there is a natural holomorphic action of $F_{V}$ on $N_{V}$.

The action is constructed as follows. Let $u$ and $\nu$ be $C^{\infty}$ sections of $F_{V}$ and $N_{V}$, respectively. Take sections $\tilde{u}$ of $F$ and $\tilde{v}$ of $T M$ so that $\left.\tilde{u}\right|_{V}=u$ and $\pi\left(\left.\tilde{v}\right|_{V}\right)=\nu$, where $\left.\right|_{V}$ means the restriction as sections. Define

$$
\alpha: \mathcal{E}^{0}\left(V, F_{V}\right) \times \mathcal{E}^{0}\left(V, N_{V}\right) \longrightarrow \mathcal{E}^{0}\left(V, N_{V}\right) \quad \text { by } \alpha(u, \nu)=\pi\left(\left.[\tilde{u}, \tilde{v}]\right|_{V}\right) .
$$

Then it is a well-defined action, which is referred to as the Camacho-Sad action.

Corollary 6.9 In the above situation, let $\nabla$ be an $F_{V}$-connection for $N_{V}$, which is also Hermitian with respect to some Hermitian metric on $N_{V}$. Then, for an invariant polynomial $\varphi$ homogeneous of degree $k>d-p$, we have $\varphi(\nabla)=0$.

\section{Localization and Hermitian residues}

We briefly recall singular holomorphic distributions, for which we refer to [24] for details. Then we prove a residue theorem for vector bundles admitting a Hermitian connection compatible with an action of the non-singular part of a singular distribution.

Singular holomorphic distributions : Let $M$ be a complex manifold of dimension $n$. We denote by $\mathcal{O}_{M}$ and $\Theta_{M}$ its structure sheaf and the tangent sheaf. For simplicity we assume that $M$ is connected. A singular distribution on $M$ is a coherent subsheaf $\mathcal{F}$ of $\Theta_{M}$. We set

$$
S(\mathcal{F})=\left\{x \in M \mid\left(\Theta_{M} / \mathcal{F}\right)_{x} \text { is not } \mathcal{O}_{M, x} \text {-free }\right\}
$$

and call it the singular set of $\mathcal{F}$. Away from $S(\mathcal{F}), \mathcal{F}$ is a locally free $\mathcal{O}$-module and its rank is called the dimension of $\mathcal{F}$. For instance, if $\mathcal{F}$ is generated by a single vector field $v, S(\mathcal{F})$ is the set of zeros of $v$.

If $\mathcal{F}$ is involutive, i.e., if $[\mathcal{F}, \mathcal{F}] \subset \mathcal{F}$, then $\mathcal{F}$ is called a singular foliation. 
The residue theorem: Let $\mathcal{F}$ be a singular distribution of dimension $p$ on $M$ with singular set $S=S(\mathcal{F})$. There is a rank $p$ subbundle $F_{0}$ of $\left.T M\right|_{U_{0}}, U_{0}=M \backslash S$, so that $\left.\mathcal{F}\right|_{U_{0}}=\mathcal{O}_{U_{0}}\left(F_{0}\right)$, the sheaf of holomorphic sections of $F_{0}$. Letting $U_{1}$ be a neighborhood of $S$, we consider the covering $\mathcal{U}=\left\{U_{0}, U_{1}\right\}$ of $M$. Let $E$ be a holomorphic vector bundle on $M$ with an action of $F_{0}$ on $U_{0}$. Let $\nabla_{0}$ be an $F_{0}$-connection for $E$ on $U_{0}$ and suppose there exists a Hermitian metric $h_{0}$ of $E$ on $U_{0}$ such that $\nabla_{0}$ is also an $h_{0}$-connection. Take a Hermitian metric $h_{1}$ of $E$ on $U_{1}$ and let $\nabla_{1}$ be the $h_{1}$-metric connection for $E$ on $U_{1}$. Recall that for an invariant polynomial $\varphi$ homogeneous of degree $k$, the characteristic class $\varphi_{\mathrm{BC}}(E)$ in $H_{\mathrm{BC}}^{k, k}(M) \simeq H_{\mathrm{BC}}^{k, k}(\mathcal{U})$ is represented by the cocycle $\varphi\left(h_{*}\right)$ in $\mathcal{E}_{\mathrm{BC}}^{k, k}(\mathcal{U})$ given by (5.9). If $k>n-p$, then by Corollary $6.4, \varphi\left(h_{0}\right)=\varphi\left(\nabla_{0}\right)=0$ and $\varphi\left(h_{*}\right)$ is expressed as

$$
\varphi\left(h_{*}\right)=\left(0, \varphi\left(h_{1}\right), \varphi\left(h_{0}, h_{1}\right)\right)
$$

so that it is in $\mathcal{E}_{\mathrm{BC}}^{k, k}\left(\mathcal{U}, U_{0}\right)$. Thus it defines a class in $H_{\mathrm{BC}}^{k, k}\left(\mathcal{U}, U_{0}\right)$, which we denote by $\varphi_{\mathrm{BC}}(E ; \mathcal{F})$ and call the localization of $\varphi_{\mathrm{BC}}(E)$ by $\mathcal{F}$ at $S$. It is sent to the class $\varphi_{\mathrm{BC}}(E)$ by the canonical morphism

$$
j^{*}: H_{\mathrm{BC}}^{k, k}\left(\mathcal{U}, U_{0}\right) \longrightarrow H_{\mathrm{BC}}^{k, k}(M) .
$$

Remark 7.1 1. The localization $\varphi_{\mathrm{BC}}(E ; \mathcal{F})$ above depends a priori on the metric $h_{0}$. Let $h_{0}^{\prime}$ be another metric of $E$ on $U_{0}$ and $\nabla_{0}^{\prime}$ the $h_{0}^{\prime}-F_{0}$-connection for $E$ on $U_{0}$. For the usual proof of the independence of the localization (cf. [21, Ch. III, Lemma 3.1]), we need to have the vanishing of $\varphi\left(h_{0}, h_{0}^{\prime}\right)$, however this is not the case in general (cf. Remark 6.5.1). On the contrary the localization is independent of the choice of the metric $h_{1}$ on $U_{1}$, for a fixed $h_{0}$ (cf. the second half of the proof of Proposition 5.10).

2. In the above situation we have the "Atiyah localization" $\varphi^{\mathrm{A}}(E ; \mathcal{F})$ in $H_{\bar{\vartheta}}^{k, k}\left(\mathcal{U}, U_{0}\right)$ (cf. [2]). It is represented by the cocycle $\left(0, \varphi^{\mathrm{A}}\left(\nabla_{1}\right), \varphi^{\mathrm{A}}\left(\nabla_{0}, \nabla_{1}\right)\right)$, which is equal to $\left(0, \varphi\left(\nabla_{1}\right), \varphi\left(\nabla_{0}, \nabla_{1}\right)\right)$ in this case (cf. Proposition 5.5 and Remark 5.61 ).

Suppose $S$ is compact. Then the image of $\varphi_{\mathrm{BC}}(E ; \mathcal{F})$ by the morphism (cf. (4.15))

$$
\Psi: H_{\mathrm{BC}}^{k, k}\left(\mathcal{U}, U_{0}\right) \longrightarrow H_{\mathrm{A}}^{n-k, n-k}[S]^{*}
$$

is denoted by $\operatorname{Res}_{\varphi_{\mathrm{BC}}}(\mathcal{F}, E ; S)$ and called the residue of $\mathcal{F}$ for $E$ at $S$ with respect to $\varphi$. If $S$ has a finite number of connected components $\left(S_{\lambda}\right)$, we take an open neighborhood $U_{\lambda}$ of $S_{\lambda}$ in $U_{1}$ for each $\lambda$ so that $U_{\lambda} \cap U_{\mu}=\emptyset$ if $\lambda \neq \mu$. Then we have the residue $\operatorname{Res}_{\varphi_{\mathrm{BC}}}\left(\mathcal{F}, E ; S_{\lambda}\right)$ in $H_{\mathrm{A}}^{n-k, n-k}\left[S_{\lambda}\right]^{*}$ for each $\lambda, H_{\mathrm{A}}^{n-p, n-q}\left[S_{\lambda}\right]=\underset{U_{\lambda} \supset S_{\lambda}}{\lim } H_{\mathrm{A}}^{n-p, n-q}\left(U_{\lambda}\right)$.

Let $R_{\lambda}$ be a $2 n$-dimensional manifold with $C^{\infty}$ boundary in $U_{\lambda}$ containing $S_{\lambda}$ in its interior and set $R_{0 \lambda}=-\partial R_{\lambda}$. Then the residue $\operatorname{Res}_{\varphi_{\mathrm{BC}}}\left(\mathcal{F}, E ; S_{\lambda}\right)$ is represented by a functional (cf. (4.8) and (4.10))

$$
\xi \mapsto \int_{R_{\lambda}} \varphi\left(h_{1}\right) \wedge \xi+\int_{R_{0 \lambda}}\left(\left((\partial-\bar{\partial}) \varphi\left(h_{0}, h_{1}\right)\right) \wedge \xi-\frac{1}{2}(\partial-\bar{\partial})\left(\varphi\left(h_{0}, h_{1}\right) \wedge \xi\right)\right)
$$

for every $\bar{\partial} \partial$-closed $(n-k, n-k)$-form $\xi$ in a neighborhood of $S_{\lambda}$.

From the above considerations and Proposition 4.16, we have the following: 
Theorem 7.3 Let $M, \mathcal{F}$ and $S$ be as above. Suppose $S$ is a compact set with a finite number of connected components $\left(S_{\lambda}\right)_{\lambda}$. Let $E$ be a holomorphic vector bundle on $M$. Assume that there is an action of $F_{0}$ on $E$ and that there is a Hermitian $F_{0}$-connection for $E$ on $U_{0}$. Let $\varphi$ be an invariant polynomial homogeneous of degree $k>n-p$. Then:

(1) for each $\lambda$ we have the residue $\operatorname{Res}_{\varphi_{\mathrm{BC}}}\left(\mathcal{F}, E ; S_{\lambda}\right)$ in $H_{\mathrm{A}}^{n-k, n-k}\left[S_{\lambda}\right]^{*}$, which is represented by the functional (7.2),

(2) if moreover $M$ is compact,

$$
\sum_{\lambda}\left(i_{\lambda}\right)_{*} \operatorname{Res}_{\varphi_{\mathrm{BC}}}\left(\mathcal{F}, E ; S_{\lambda}\right)=\Phi\left(\varphi_{\mathrm{BC}}(E)\right) \quad \text { in } H_{\mathrm{A}}^{n-k, n-k}(M)^{*}
$$

where $i_{\lambda}: S_{\lambda} \hookrightarrow M$ denotes the inclusion.

A residue as $\operatorname{Res}_{\varphi_{\mathrm{BC}}}\left(\mathcal{F}, E ; S_{\lambda}\right)$ is referred to as a Hermitian residue.

Remark 7.4 If $k=n$ and if $M$ is compact and connected, $H_{\mathrm{A}}^{n-k, n-k}(M)^{*}=H_{\mathrm{A}}^{0,0}(M)^{*}$ may be identified with $\mathbb{C}$, and in this case, $\left(i_{\lambda}\right)_{*} \operatorname{Res}_{\varphi_{\mathrm{BC}}}\left(\mathcal{F}, E ; S_{\lambda}\right)$ is a complex number given by

$$
\int_{R_{\lambda}} \varphi\left(h_{1}\right)+\frac{1}{2} \int_{R_{0 \lambda}}(\partial-\bar{\partial}) \varphi\left(h_{0}, h_{1}\right),
$$

and $\Phi\left(\varphi_{\mathrm{BC}}(E)\right)$ may be expressed as $\int_{M} \varphi_{\mathrm{BC}}(E)$.

\section{An example}

For $\lambda \in \mathbb{C}^{*}$ with $|\lambda|<1$, we consider the Hopf surface $V=\left(\mathbb{C}^{2} \backslash\{0\}\right) / \sim$, where $(x, y) \sim(\lambda x, \lambda y)$. There is a fibration $\rho: V \rightarrow \mathbb{P}^{1}$ by elliptic curves. Let $L$ denote the pull-back by $\rho$ of the hyperplane bundle on $\mathbb{P}^{1}$. Then, as $H_{d}^{2}(V)=0$ and $H_{\bar{\partial}}^{1,1}(V)=0$, both the Chern class $c^{1}(L)$ and the Atiyah class $a^{1}(L)$ vanish. On the other hand, $H_{\mathrm{BC}}^{1,1}(V) \simeq \mathbb{C}$ (cf. [3]) and the Bott-Chern class $c_{\mathrm{BC}}^{1}(L)$ is a generator. We show that it is localized at one of the fibers $C$ of $\rho$. For this we realize $V$ as an invariant submanifold of a singular foliation on an ambient manifold whose singular set on $V$ is $C$. Then the Camacho-Sad action of the foliation on $L$ away from $C$ gives the localization (cf. Subsection 6.2).

We consider the Hopf manifold $M=\left(\mathbb{C}^{3} \backslash\{0\}\right) / \sim$, where $(x, y, z) \sim(\lambda x, \lambda y, \lambda z)$. Let $\mathcal{F}$ be the two-dimensional foliation on $M$ induced by the vector fields

$$
v_{1}=y \frac{\partial}{\partial y}+z \frac{\partial}{\partial z} \quad \text { and } \quad v_{2}=\frac{\partial}{\partial x}
$$

which leaves invariant the Hopf surface $V=\{z=0\} / \sim \subset M$. The $\operatorname{singular}$ set $S(\mathcal{F})$ of $\mathcal{F}$ is given by

$$
S(\mathcal{F})=C=\{y=z=0\} / \sim \subset V .
$$

There is a fibration $\tilde{\rho}: M \rightarrow \mathbb{P}^{2}$ by elliptic curves. It restricts to the fibration $\rho: V \rightarrow \mathbb{P}^{1}$, of which $C$ is a fiber. Recall the exact sequence (6.6):

$$
\left.0 \longrightarrow T V \longrightarrow T M\right|_{V} \stackrel{\pi}{\longrightarrow} N_{V} \longrightarrow 0
$$


If we denote by $\tilde{L}$ the pull-back of the hyperplane bundle on $\mathbb{P}^{2}$ by $\tilde{\rho}$, we have $L=\left.\tilde{L}\right|_{V}$. The normal bundle of $V$ is given by $N_{V}=L$, since $T M=\tilde{L} \oplus \tilde{L} \oplus \tilde{L}$ and $T V=L \oplus L$.

We try to localize $c_{\mathrm{BC}}^{1}\left(N_{V}\right)$ on $C$. Denoting by $[x, y]$ the image of $(x, y)$ by the canonical surjection $\mathbb{C}^{2} \backslash\{0\} \rightarrow V$, we set $U_{0}=\{[x, y] \mid y \neq 0\}$ and $U_{1}=\{[x, y] \mid x \neq 0\}$ and consider the covering $\mathcal{U}=\left\{U_{0}, U_{1}\right\}$ of $V$. We have that $V \backslash C=U_{0}$ and $C \subset U_{1}$. The bundle $L$ is described as

$$
L=\left(U_{0} \times \mathbb{C}\right) \cup\left(U_{1} \times \mathbb{C}\right),
$$

where $\left([x, y], \zeta_{0}\right)$ and $\left([x, y], \zeta_{1}\right)$ are identified if and only if $\zeta_{0}=x / y \cdot \zeta_{1}$.

Also, if we set $s=x / y$ on $U_{0}, s$ is a base coordinate and $y$ is a (covering) fiber coordinate of the fibration $\rho: V \rightarrow \mathbb{P}^{1}$ and, if we set $t=y / x$ on $U_{1}, t$ is a base coordinate and $x$ is a fiber coordinate of the fibration $\rho$.

The foliation $\mathcal{F}$ defines a subbundle $F_{0}$ of $\left.T V\right|_{U_{0}}$ of rank 2 and there is the CamachoSad action of $F_{0}$ on $\left.N_{V}\right|_{U_{0}}$. Let $\nabla$ be an $F_{0}$-connection for $\left.N_{V}\right|_{U_{0}}$ and we compute the connection form for $\nabla$ (cf. Definition 6.2 and (6.8)). The connection is uniquely determined in our case and turns out to be Hermitian.

We compute the connection form locally and find that the expression is valid globally on $U_{0}$. At each point of $U_{0},(x, y, z)$ is a coordinate system on $M$ in a neighborhood $\tilde{W}$ of the point. We set $W=V \cap \tilde{W}$. We take $\nu=\pi(\partial / \partial z)$ as a holomorphic frame of $N_{V}$ on $W$ and let $\theta$ be the connection form of $\nabla$ with respect to $\nu$. Since $\theta$ is of type $(1,0)$, we may write $\theta=f d x+g d y$. Thus $\nabla_{v_{1}}(\nu)=y g \nu$ and $\nabla_{v_{2}}(\nu)=f \nu$. On the other hand, we compute

$$
\nabla_{v_{1}}(\nu)=\pi\left(\left.\left[y \frac{\partial}{\partial y}+z \frac{\partial}{\partial z}, \frac{\partial}{\partial z}\right]\right|_{W}\right)=-\nu \quad \text { and } \quad \nabla_{v_{2}}(\nu)=\pi\left(\left.\left[\frac{\partial}{\partial x}, \frac{\partial}{\partial z}\right]\right|_{W}\right)=0 .
$$

Thus we conclude that

$$
\theta=-\frac{d y}{y}=-\partial \log |y|^{2}
$$

Note that the expression is a priori on $W$, however it is valid on the whole $U_{0}$. Moreover, this shows that $\nabla$ is Hermitian. In fact, on $U_{0},[x, y] \mapsto \zeta_{0}=1 / y$ is a frame defining a connection form $-\partial \log |y|^{2}$ with respect to the standard Hermitian metric on $\left.L\right|_{U_{0}} \simeq$ $U_{0} \times \mathbb{C}$ (cf. (5.3), Remark 5.6.2). In this case, we can directly verify the vanishing theorem (Corollary 6.4) as

$$
c^{1}(\nabla)=\frac{\sqrt{-1}}{2 \pi} \bar{\partial} \theta=-\frac{\sqrt{-1}}{2 \pi} \bar{\partial} \partial \log |y|^{2}=0 .
$$

Now, also we take on $U_{1}$ the standard metric and consider $1 / x$ as a non-vanishing section of $N_{V}=L$ on $U_{1}$. Then, setting $\gamma=\frac{1}{2 \pi \sqrt{-1}}$, the class $c_{\mathrm{BC}}^{1}\left(N_{V}\right)$ is represented by the cocycle (cf. Remark 5.9.3)

$$
\gamma\left(\bar{\partial} \partial \log |y|^{2}, \bar{\partial} \partial \log |x|^{2}, \log |x / y|^{2}\right)=\left(0,0, \gamma \log |x / y|^{2}\right)=-\left(0,0, \gamma \log |t|^{2}\right)
$$

and it defines the localization $c_{\mathrm{BC}}^{1}(L ; \mathcal{F}) \in H_{\mathrm{BC}}^{1,1}\left(\mathcal{U}, U_{0}\right)$ of $c_{\mathrm{BC}}^{1}(L)$.

Now we examine its residue $\operatorname{Res}_{c_{\mathrm{BC}}^{1}}(\mathcal{F}, L ; C) \in H_{\mathrm{A}}^{1,1}[C]^{*}$. We have the following diagram, of which the square part is commutative (cf. Proposition 4.16):

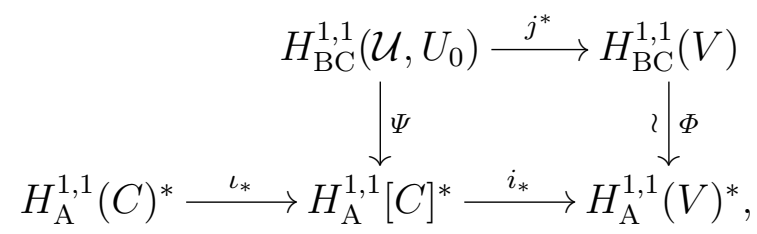


where $\iota_{*}$ is the transpose of the restriction $\iota^{*}: H_{\mathrm{A}}^{1,1}[C] \rightarrow H_{\mathrm{A}}^{1,1}(C)$. We have $H_{\mathrm{A}}^{1,1}(C) \simeq \mathbb{C}$ and the composition $\iota^{*} \circ i^{*}: H_{\mathrm{A}}^{1,1}(V) \rightarrow H_{\mathrm{A}}^{1,1}(C)$ is an isomorphism, however note that $H_{\mathrm{A}}^{1,1}[C]$ is infinite dimensional.

The residue $\operatorname{Res}_{c_{\mathrm{BC}}^{1}}(\mathcal{F}, L ; C)$ is the functional that assigns to each $\bar{\partial} \partial$-closed $(1,1)$-form $\xi$ in a neighborhood of $C$ the value (cf. (7.2))

$$
-\gamma \int_{R_{01}}\left((\partial \log t-\bar{\partial} \log \bar{t}) \wedge \xi-\frac{1}{2}(\partial-\bar{\partial})\left(\log |t|^{2} \cdot \xi\right)\right)
$$

where $R_{1}=\left\{(t, x) \in U_{1}|| t \mid \leq \delta\right\}$ for some $\delta>0$ and $R_{01}=-\partial R_{1}$.

The canonical generator of $H_{\mathrm{A}}^{1,1}(C)=\mathbb{C}$ is given by

$$
\xi_{0}=\frac{\gamma}{\log |\lambda|} d \log x \wedge d \log \bar{x}
$$

It may be thought of as representing the class in $H_{\mathrm{A}}^{1,1}(V)$ dual to $c_{\mathrm{BC}}^{1}(L)$, which is represented by $\gamma \bar{\partial} \partial \log \left(|x|^{2}+|y|^{2}\right)$, in $H_{\mathrm{BC}}^{1,1}(V)$. Now we calculate $\operatorname{Res}_{c_{\mathrm{BC}}^{1}}(\mathcal{F}, L ; C)\left(\xi_{0}\right)$ (cf. (8.1)) and verify that it is equal to one. Since $\partial \xi_{0}=\bar{\partial} \xi_{0}=0, \partial\left(\log |t|^{2} \cdot \xi_{0}\right)=(\partial \log t) \wedge \xi_{0}$ and $\bar{\partial}\left(\log |t|^{2} \cdot \xi_{0}\right)=\bar{\partial}(\log \bar{t}) \wedge \xi_{0}$. Thus

$$
\operatorname{Res}_{c_{\mathrm{BC}}^{1}}(\mathcal{F}, L ; C)\left(\xi_{0}\right)=\frac{\gamma}{2} \int_{\partial R_{1}}(\partial \log t-\bar{\partial} \log \bar{t}) \wedge \xi_{0}=\int_{C} \xi_{0}=1 .
$$

\section{References}

[1] M. Abate, F. Bracci and F. Tovena, Index theorems for holomorphic self-maps, Ann. of Math. 159 (2004), 819-864.

[2] M. Abate, F. Bracci, T. Suwa and F. Tovena, Localization of Atiyah classes, Rev. Mat. Iberoam. 29 (2013), 547-578.

[3] D. Angella, G. Dloussky and A. Tomassini, On Bott-Chern cohomology of compact complex surfaces, Annali di Mat. Pura ed Applicata 195 (2016), 199-217.

[4] D. Angella and A. Tomassini, On the $\partial \bar{\partial}-$ Lemma and Bott-Chern cohomology, Invent. Math. 192 (2013), 71-81.

[5] P. Baum and R. Bott, Singularities of holomorphic foliations, J. Differential Geom. 7 (1972), 279-342.

[6] J.-M. Bismut, H. Gillet and C. Soulé, Analytic torsion and holomorphic determinant bundles I: Bott-Chern forms and analytic torsion, Comm. Math. Phys. 115 (1988), 49-78.

[7] R. Bott, Lectures on characteristic classes and foliations, Lectures on Algebraic and Differential Topology, Lecture Notes in Math. 279, Springer 1972, 1-94.

[8] R. Bott and S. S. Chern, Hermitian vector bundles and the equidistribution of the zeroes of their holomorphic sections, Acta Math. 114 (1968), 71-112. 
[9] R. Bott and L. Tu, Differential Forms in Algebraic Topology, Graduate Texts in Mathematics 82, Springer, 1982.

[10] J.-P. Brasselet, J. Seade and T. Suwa, Vector Fields on Singular Varieties, Lecture Notes in Math. 1987, Springer 2009.

[11] J.I. Burgos Gil, J. Kramer and U. Kühn, Arithmetic characteristic classes of automorphic vector bundles, Documenta Math. 10 (2005), 619-716.

[12] J.I. Burgos Gil and R. Liţkanu, Singular Bott-Chern classes and the arithmetic Grothendieck-Riemann-Roch theorem for closed immersions, Documenta Math. 15 (2010), 73-176.

[13] J.B. Carrell, An analytic obstruction to a complex toral action on a complex manifold, J. Differential Geom. 7 (1972), 393-401.

[14] H. Gillet and C. Soulé, Characteristic Classes for Algebraic Vector Bundles with Hermitian Metric, I, Ann. of Math. 131 (1990), 163-203.

[15] P. Griffiths and J. Harris, Principles of Algebraic Geometry, John Wiley \& Sons, 1978.

[16] D. Lehmann, Systèmes d'alvéoles et intégration sur le complexe de Čech-de Rham, Publications de l'IRMA, 23, No VI, Université de Lille I, 1991.

[17] D. Lehmann, Résidus des sous-variétés invariantes d'un feuilletage singulier, Ann. Inst. Fourier 41 (1991), 211-258.

[18] D. Lehmann and T. Suwa, Residues of holomorphic vector fields relative to singular invariant subvarieties, J. Differential Geom. 42 (1995), 165-192.

[19] J. Milnor and J. Stasheff, Characteristic Classes, Ann. of Math. Studies 76, Princeton University Press, 1974.

[20] M. Schweitzer, Autour de la cohomologie de Bott-Chern, arXiv:0709.3528v1.

[21] T. Suwa, Indices of Vector Fields and Residues of Singular Holomorphic Foliations, Actualités Mathématiques, Hermann Paris, 1998.

[22] T. Suwa, Residue Theoretical Approach to Intersection Theory, Real and Complex Singularities, Contemporary Math., American Math. Soc. 459 (2008), 207-261.

[23] T. Suwa, Čech-Dolbeault cohomology and the $\bar{\partial}$-Thom class, Advanced Studies in Pure Math. 56, 2009, 321-340.

[24] T. Suwa, Residues of singular holomorphic distributions, Singularities in Geometry and Topology Strasbourg 2009, IRMA Lectures in Mathematics and Theoretical Physics 20, European Math., Soc. 2012, 207-247.

[25] T. Suwa, Representation of relative sheaf cohomology, arXiv:1810.06198.

[26] T. Suwa, Relative Dolbeault cohomology, arXiv:1903.04710. 
M. Corrêa

Departamento de Matemática

Universidade Federal de Minas Gerais

Av. Antônio Carlos 6627

30161-970 Belo Horizonte, Brazil

mauricio@mat.ufmg.br

T. Suwa

Department of Mathematics

Hokkaido University

Sapporo 060-0810, Japan

tsuwa@sci.hokudai.ac.jp 